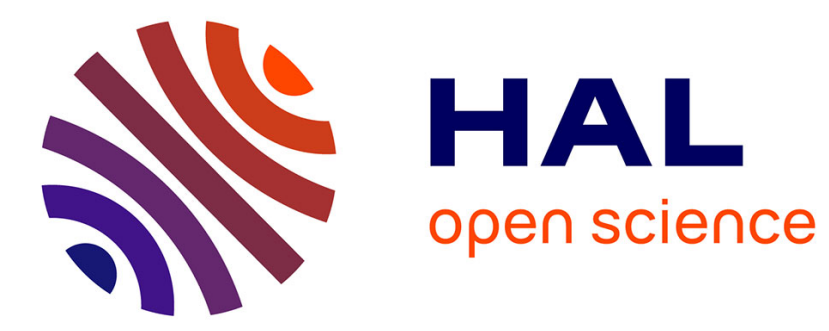

\title{
Electronic properties of chemically doped graphene
}

Frédéric Joucken, Luc Henrard, Jérôme Lagoute

\section{To cite this version:}

Frédéric Joucken, Luc Henrard, Jérôme Lagoute. Electronic properties of chemically doped graphene. Physical Review Materials, 2019, 3 (11), 10.1103/PhysRevMaterials.3.110301 . hal-02361622

\section{HAL Id: hal-02361622 \\ https://hal.science/hal-02361622}

Submitted on 8 Nov 2020

HAL is a multi-disciplinary open access archive for the deposit and dissemination of scientific research documents, whether they are published or not. The documents may come from teaching and research institutions in France or abroad, or from public or private research centers.
L'archive ouverte pluridisciplinaire HAL, est destinée au dépôt et à la diffusion de documents scientifiques de niveau recherche, publiés ou non, émanant des établissements d'enseignement et de recherche français ou étrangers, des laboratoires publics ou privés. 


\title{
Electronic properties of chemically-doped graphene
}

\author{
Frédéric Joucken \\ Department of Physics, University of California, Santa Cruz, California 95060, USA* \\ Luc Henrard \\ Department of Physics and Namur Institute of Structured Materials, \\ University of Namur, Rue de Bruxelles 51, 5000 Namur, Belgium \\ Jérôme Lagoute \\ Laboratoire Matériaux et Phénomènes Quantiques, UMR7162, \\ Université Paris Diderot Paris 7, Sorbonne Paris Cité, CNRS, \\ UMR 7162, Case courrier 7021, 75205 Paris 13, France
}

(Dated: 5 novembre 2019)

\begin{abstract}
Chemical doping of graphene is the most robust way of modifying graphene's electronic properties. We review here the results obtained so far on the electronic structure and transport properties of chemically-doped graphene, focusing on the results obtained with scanning tunneling micropscopy/spectroscopy (STM/S), angle-resolved photoemission spectroscopy (ARPES), and magnetoresistance (MR) measurements. The majority of the results reported have been obtained on nitrogendoped samples, but boron-doped graphene has also been well documented. Besides the appearance of the dopant on STM topographic images, the main questions that have been addressed are the atomic configurations of the doping and their doping efficiency (number of electron/hole brought to the graphene lattice). Both can be addressed by a local probe such as STM/S. The doping efficiency has also been complementary studied via direct visualization of the band structure with ARPES. The effect of the dopants on the electronic transport properties and in particular their influence on the scattering mechanisms is also presented. Finally, avenues for future research efforts are suggested.
\end{abstract}

\section{INTRODUCTION}

The controlled modification of graphene's electronic structure through chemical doping has been pursued for several years. The motivations behind these investigations are both applied and fundamental. Doped graphene has been envisioned for various applications, such as electrochemical biosensing [1, 2], metal free electrocatalysis $[3,4]$, lithium ion battery [5-7], or supercapacitors [8, 9]. Controlled $n$ - or $p$-doping of graphene will also allow synthesize robust $p$ - $n$ lateral junctions [10] or vertical heterostructures [11] for e.g. photodetection applications [12-14]. On the fundamental side, the tunneling spectroscopic signature of the dopants, the free charge carrier passed on to the graphene lattice by the dopants, the role of local electronic states on optical and transport properties, the screening of local charge defect in $2 \mathrm{D}$ or their influence on the various scattering mechanisms in electronic transport are important questions to unravel.

Unlike previous reviews on doped graphene [15-21], our purpose is to focus on the progress made specifically on the dopants effects on the electronic structure and the electronic transport properties of graphene. By "chemical doping" we mean the substitution of one or several carbon atoms by other chemical elements in the graphene lattice, possibly accompanied by a local lattice modification, such as a vacancy. We will show that, although

\footnotetext{
* frederic.joucken@gmail.com
}

important progress has been made, some important issues require further investigation. The discussion is focused here on graphene but is also of relevance for other two-dimensional systems, such as transition metal dichalcogenides or black phosphorus, where chemical doping is at its infancy.

The investigation of the structural and electronic properties of modified graphene are based on many experimental techniques. In this review, we put forward three of them that give very specific information for graphene-related materials : scanning tunneling microscopy/spectroscopy (STM/S), angle-resolved photoemission spectropscopy (ARPES), and magnetoresistance (MR) measurements. The first one provides local information on the electronic structure at the atomic scale while the two others probe the global properties of the materials. The input of other experimental techniques is also included but without dedicated section. Interpretation of the experimental data is often based on density functional theory (DFT) and tight-binding (TB) simulations so that we also discuss these theoretical results.

Mainly because of its proximity to carbon in the periodic table, its similar size, and the easiness with which it is manipulated, nitrogen $(\mathrm{Z}=7)$ is by far the most studied dopant so that this review mainly discusses results obtained for this element. We already note here that, in analogy with semiconductor, nitrogen naturally behaves like an electron donor when it substitutes carbon in a lattice. However, it might also trap electron in other atomic configuration like pyridine-like so that the comparison with bulk semiconductor like $\mathrm{Si}$ or GaAs is not straight- 
forward. Boron $(\mathrm{Z}=5)$ is the second most studied dopant, because also of its proximity to carbon in the periodic table and of its expected acceptor behavior. Chemical doping with other elements such as sulphur [22-24], phosphorus [25-28], or fluorine [29, 30] has also been reported and considered for various applications, but electronic structure or MR measurements on graphene doped with elements other than nitrogen and boron are still missing.

The structure of the article is the following. As preliminary, we describe the various configurations dopants can adopt in graphene honeycomb lattice and the various ways of synthesizing chemically-doped graphene. We also discuss briefly the basics of graphene electronic structure. We then review the literature results obtained on chemically-doped graphene with the three different experimental techniques we chose to focus on (STM/S, ARPES, and MR), in separate sections. For each technique, results for nitrogen and boron dopants are presented. We conclude our review by pointing out open questions which future efforts could tackle.

\section{PRELIMINARY}

\section{A. Atomic structure}

The dopants can be embedded in the graphene lattice in various configurations (Fig. 1). For single dopant, the most simple configuration (and the most stable) is the single substitution (or 'graphitic' configuration), where the dopant replace a single carbon atom in the graphene lattice. The 'pyridinic' and the 'pyrrolic' configurations include a vacancy and are also often observed. 'Pyridinic' configuration is associated to two atomic configurations (3N- and N-pyridinic) depending on the number of $\mathrm{N}$ atoms involved. When dopants are close to each other, their mutual interactions modify their electronic spectrum and these configurations are therefore considered as nitrogen pairs. Different types of pairs have been observed and can be defined using the nomenclature used in ref. [31], where pairs are simply referred by numbering the atomic site where the dopant is present.

\section{B. Synthesis}

Two routes can be taken for the synthesis of doped graphene : either doping is achieved during the graphene synthesis (direct doping) or after the graphene growth, (post-growth doping). For the papers reviewed here, the most common technique for direct doping is chemical vapor deposition (CVD) growth (such as in refs. [32-34]), while plasma-based ions (or radicals) bombardment is mainly used for post-growth doping (such as in refs. [3538]). As expected and as discussed extensively below, the doping method influences dramatically the doping results (such as the dominant dopant configuration or the occurrence of sublattice segregation).

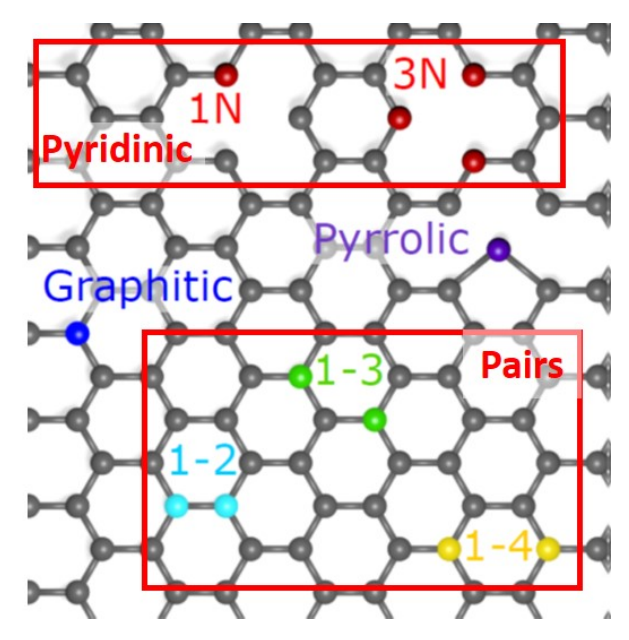

FiguRE 1. Common atomic configurations of nitrogen dopants in the graphene lattice. Illustrated are the simple and triple pyridinic, the pyrrolic, and the graphitic (or substitutional) configurations, as well as common pairs.

\section{Electronic properties of doped graphene}

Graphene hosts massless Dirac-like electronic excitations, with the valence and conduction band Dirac cones crossing at the Dirac energy $\left(E_{D}\right)$ at the $\mathrm{K}$ point of the Brillouin Zone [39]. For neutral graphene the Fermi energy $\left(E_{F}\right)$ is located at the Dirac energy. As illustrated in Fig. 2a, the density of electronic states is zero at $E_{D}$ and then increases linearly for small energy difference. This means that adding an extra electron density $n$ to the graphene sheet will shift the Fermi energy away from the Dirac energy (Fig. 2b, dashed line) such that

$$
n=\frac{\left(\hbar v_{F}\right)^{2}}{\pi}\left(E_{D}-E_{F}\right)^{2}
$$

$\left(v_{F}\right.$ is the Fermi velocity). Besides electrostatic doping [40], the chemical substitution of some carbon atoms by foreign atoms is a manner to change the charge carrier concentration (Fig. 2b, solid line). The most natural dopants, nitrogen and boron, are expected to lead to electron and hole doping, respectively. The intuitive picture is that extra valence electrons/holes of the substitutional atoms are transferred to the graphitic network. However, this view has to be moderated as only a fraction of these 'extra' electrons/holes are involved in this process and states localized around the dopant also appear (Fig. 2b, solid line) $[32,35]$, as discussed later in this review. Other doping configuration results in different electronic signature as exemplified by the shift of the Fermi level associated with p-doping obtained for the pyridine configuration in the case of nitrogen [41]. Short range and long range interaction between dopant also influence the electronic properties [31, 42], as discussed later.

It is important at this point to comment on the analogy between the substitutional doping in graphene and in $3 \mathrm{D}$ semiconductors such as silicon. The common point is 

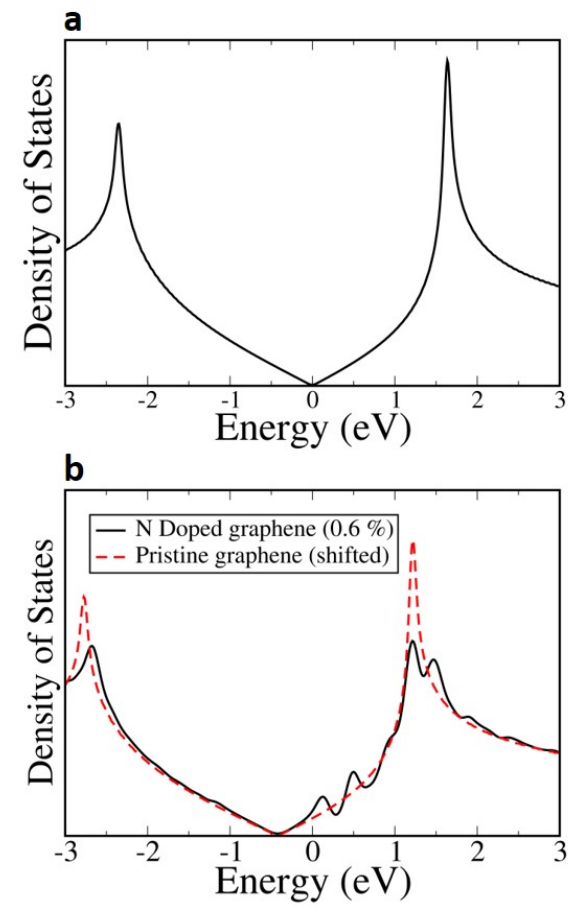

FIGURE 2. Density of states (DOS) of graphene computed by DFT. (a) For pristine graphene. (b) For N-Doped graphene with 0.6 at. $\%$ of nitrogen atoms (solid line). The dashed curve reproduces the DOS of the pristine graphene shifted by $0.42 \mathrm{eV}$. From [35].

the substitution of quadrivalent atoms by pentavalent or trivalent ones to obtain $n$-doped or $p$-doped compound. However, in 3D traditional semiconductors, the localized acceptor or donnor states lie in the electronic band gap and thermal activation allows to populate the conduction band with electrons and the valence band with holes, the relative dopant density being on the order of $10^{-6}$ to $10^{-10}$. On the other side, graphene does not have a band gap and extra electrons and holes are associated to a shift of the Fermi level of the gapless electronic density, with typical dopant concentration much higher than for 3D semiconductors (tenths of at. \% to several at. \%).

\section{SCANNING TUNNELING MICROSCOPY AND SPECTROSCOPY}

\section{Inelastic tunneling into graphene}

We start this section by recalling the peculiar aspects of electron tunneling into graphene. Zhang et al. have shown that when an STM tip is calibrated against the Shockley surface state of a $\mathrm{Au}(111)$ surface, the tunneling spectrum of graphene is dominated by an inelastic channel above a certain tip-sample bias threshold $(\sim 65 \mathrm{meV})$ [43]. The main signature of the inelastic contribution is a pseudogap observed in the STS spectra, whose width corresponds to twice the energy of the phonon involved in the inelastic channel. Because the electrons lose the phonon energy in the tunneling process by creating a phonon, the energy of features of interest observed on the STS spectra (such as the Dirac point) has to be renormalized by the phonon energy. Although the inelastic signature was initially attributed to a single additional inelastic channel, it was later found that there were actually several channels involved [44-46]. The presence of several channels makes it difficult to precisely determine the energy of features of interest in the STS spectra [47]. We discuss below the influence of dopants on the inelastic nature of the tunneling in graphene (section IA) and also how the combination of ARPES and STM can be used to corroborate the interpretation of the pseudogap in STS spectra as the signature of phonon-assisted tunneling (section II A).

\section{A. Nitrogen}

\section{STM imaging and electronic doping from STS spectra}

Although the first STM images of N-doped graphene were presumably reported by Deng et al. [48], the first atomic scale electronic characterization combining imaging and spectroscopy was performed by Zhao et al. [32] (Fig. 3a and 3c). By combining STM and STS with DFT and near edge X-ray absorption fine structure spectroscopy (NEXAFS) results, they unambiguously identified the defects they imaged by STM as graphitic nitrogen. They showed that the majority of the dopants in their samples - synthesized by CVD on copper foil with a mixture of $\mathrm{CH}_{4}, \mathrm{H}_{2}$ and $\mathrm{NH}_{3}$ as precursors - adopted the same graphitic configuration and that the measured N-N distance indicates a random distribution of the dopants. By relating the measured nitrogen concentration (determined by counting the defects on STM images) to the Dirac point position relative to the Fermi level $\left(E_{D}-E_{F}\right)$ in the STS spectra (eq. 1) (Fig. 3c), they also determined a doping efficiency of 0.42 , i.e. that each $\mathrm{N}$ dopant contribute on average 0.42 carrier to the electron density.

Subsequent local spectroscopic probe investigation has revealed very similar STM imaging of the graphitic nitrogen (Fig. 3b) [35], although the sample was produced in a completely different fashion (by exposing quasifreestanding graphene grown on $\mathrm{SiC}(000 \overline{1})$ to a nitrogen radical flux produced by a remote radio-frequency plasma source in ultra-high vacuum conditions). Tunneling spectroscopy (Fig. 3d) allowed similar measurements of the electron per dopant atom but a very different value of 0.8 was found. The values for the doping efficiency deduced from STS measurements should therefore be taken cautiously. Indeed, as discussed above, the measurement of the Dirac point position by STS is plagued by the dominance of inelastic channels in the tip-graphene tunnel junction $[45,49,50]$ and precise determination of $E_{D}$ for large doping is difficult [47]. 


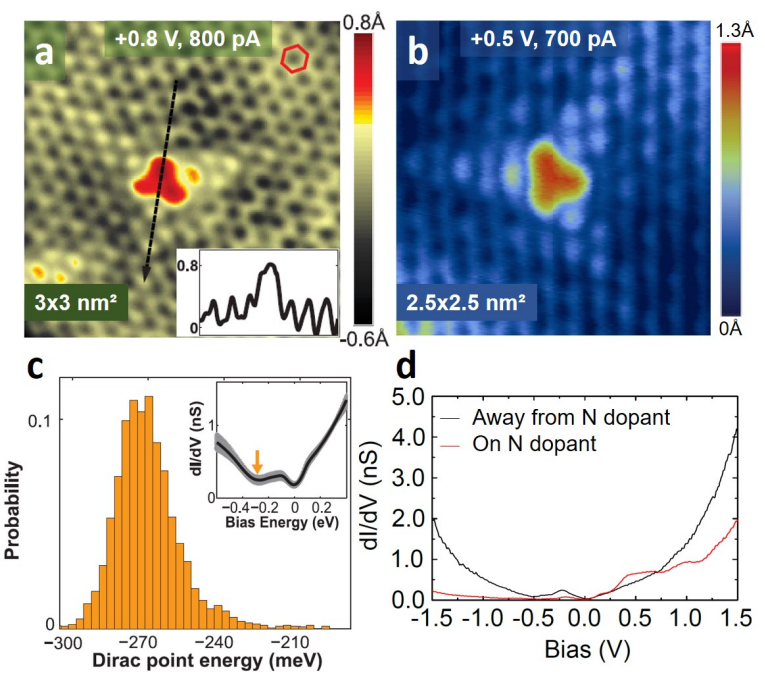

FIGURE 3. Early STM/STS characterization of graphitic nitrogen in graphene. (a) STM image of a graphitic nitrogen dopant in graphene on copper foil. (Inset) Line profile across the dopant shows atomic corrugation and apparent height of the dopant. $\mathrm{V}=0.8 \mathrm{~V}, \mathrm{I}=0.8 \mathrm{nA}$. (b) STM image of a graphitic nitrogen dopant in graphene on $\mathrm{SiC}(000 \overline{1}) . \mathrm{V}=0.5 \mathrm{~V}$, $\mathrm{I}=0.7 \mathrm{nA}$. (c) Histogram of the spatial variation of the Dirac point for $\mathrm{N}$-doped graphene on copper foil over an area of $40 \times 40 \mathrm{~nm}^{2} . \mathrm{V}=0.6 \mathrm{~V}, \mathrm{I}=1.0 \mathrm{nA}$ ). (Inset) Spatially averaged $\mathrm{dI} / \mathrm{dV}$ spectrums (black line) and variation (gray band) over the area. The orange arrow indicates the position of the Dirac point. (d) Tunneling spectra obtained on top of the nitrogen dopant (red curve) and $1.5 \mathrm{~nm}$ away (black curve). (a) and (c) from [32]. (b) and (d) from [35].

The above-mentioned first STM images reveals a triangular shape contrast centered on the $\mathrm{N}$ atom (Fig. 3), involving the neighboring $\mathrm{C}$ atoms $[32,35]$ whereas the first simulations by the Tersoff-Hamman approach predict a hollow triangular shape pattern with low intensity above the $\mathrm{N}$ atom as a result of the spatial extension of the electron density above the different atomic species [41]. Further analysis showed that depending on the imaging conditions both patterns are observed (Figs 4 and 5). According to DFT simulations, both the tip-graphene distance [31] and the composition [36] or shape of the tip [51] could explain the observations of different STM patterns for the same atomic configurations, rather than the influence of the bias. This substantial variation of the pattern from one experiment to the next was further illustrated by van der Heijden et al. by combining STM and atomic force microscopy (AFM) (Fig. 6) [52] : although the defect appears asymmetric in the STM image and is different from other reports (cf. e.g. Figs. 3 and 4), the AFM image demonstrates the dopant is in the graphitic configuration. STM imaging of graphitic nitrogen in graphene and electron doping were subsequently reported by other groups [53-62].

The relative distribution of dopants on each sublattice can be evaluated from STM images. The orientation of the triangular pattern of graphitic nitrogen de-

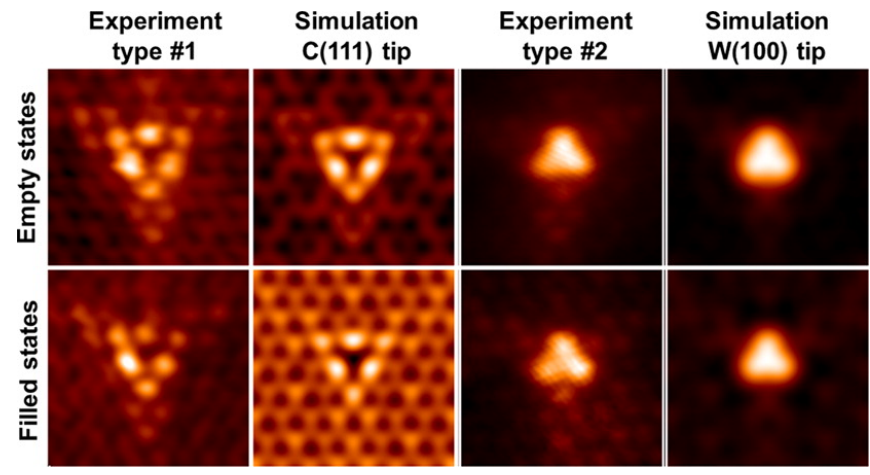

FiguRE 4. Influence of the tip's state on the STM image of graphitic nitrogen. Experimental and calculated STM current maps of the single $\mathrm{N}$ defects, empty and filled states, $1.7 \times 1.7 \mathrm{~nm}^{2}$. STM simulations $(+0.5$ and $-0.5 \mathrm{~V}$, surface-tip distance $3.5 \AA$ ) for various tip models are matched to the two most frequently experimentally observed patterns $(+0.4$ and $-0.4 \mathrm{~V})$. From [36].
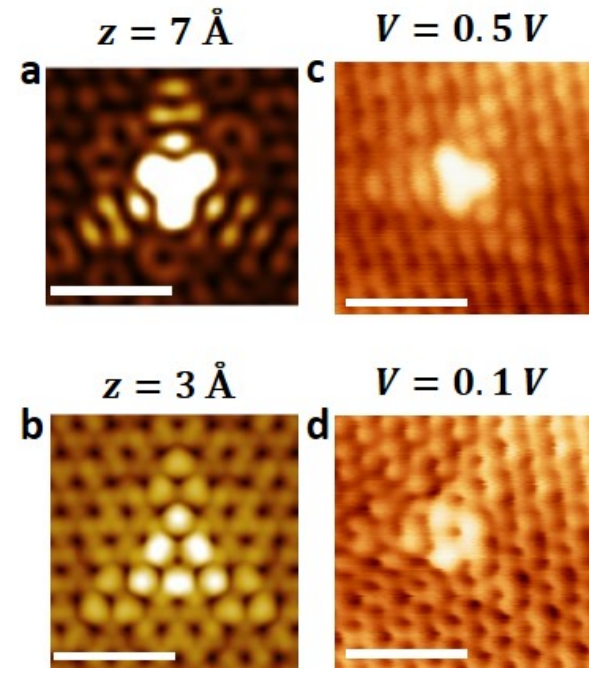

FIGURE 5. Influence of the tip-sample distance and of the bias on the STM image of graphitic nitrogen. (a) and (b) are DFT-based STM image simulations at different tip-sample distance ( 7 and $3 \AA$, respectively). Simulations with the same parametrization as in [31]. (c) and (d) are experimental STM images at different sample bias ( 0.5 and 0.1 $\mathrm{V}$, respectively). Scale bars are $1 \mathrm{~nm}$.

pends on the sublattice on which the nitrogen is located (Fig. 7a and 7b). This relative distribution has been found in certain cases to be unbalanced between the two sublattices of graphene. The unbalance was weak for samples synthesized with post-growth methods but surprisingly still present [36], and was stronger in CVDgrown samples [32]. In samples prepared on single crystals, it was found that the sublattice segregation can be almost complete, although limited to relatively small areas $\left(\sim 100 \times 100 \mathrm{~nm}^{2}\right)$ (Fig. 7c) [63]. Finally, we note that Neilson et al. reported recently grouping of graphitic nitrogen into curved lines and pyridinc nitrogen at the 


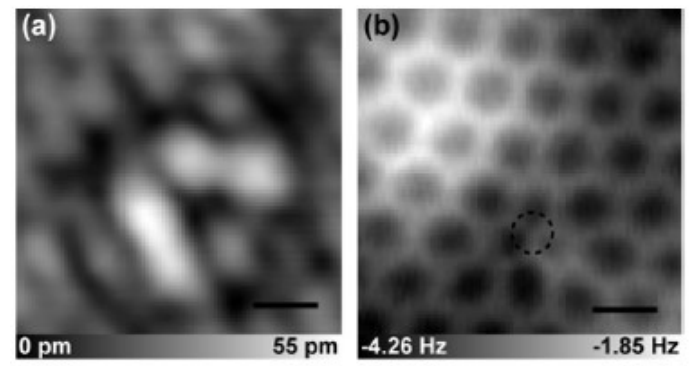

FIGURE 6. Illustration of the difficulty of identifying the atomic configuration of dopant from STM data alone. (a) STM image of a $\mathrm{N}$ dopant in graphene on $\mathrm{Cu}(111)$. $\mathrm{I}=50 \mathrm{pA}$ at $\mathrm{V}=1.0 \mathrm{~V}$. (b) Constant-height AFM image of the same location at $-20 \mathrm{pm}$ with respect to STM set point. The dopant position is indicated by the dashed circle in (b). From $[52]$.

edges of graphene islands when using the sole precursor azafullerene $\left(\mathrm{C}_{59} \mathrm{NH}\right)$ for growing $\mathrm{N}$-doped graphene on $\mathrm{Cu}(111)$ [64].

\section{Localized state and interaction between nitrogen dopants}

Early DFT calculations for graphitic nitrogen predicted a localized donor state about $1 \mathrm{eV}$ above $E_{D}$ with a double-peak signature in the density of states [41] (Fig. 2c). The unique broad feature observed experimentally by STS (red curve in Fig. 3d) [35] was first explain by an experimental broadening. Lambin et al. later showed that supercell geometries used in DFT calculations create artificial long range interaction between dopants [42]. Combining DFT with TB, they showed that the density of states peaks structure predicted in the conduction band of N-doped graphene depends on the size of the supercell used in the calculation. More interestingly, the simulation for a random distribution of the dopant can be directly compared with experimental STS [42]. In the same study on the long range interaction, they emphasized the role of symmetry of the dopant position to open a bang gap in graphene. In particular, a random distribution of dopant restricted to the same sublattice does open a band gap.

Tison et al. have combined STM/STS results with DFT and TB simulations to investigate the interaction between graphitic dopants [31]. As theoretically predicted, the localized state observed in the tunneling spectra was shown to be closer to the Dirac point for the nitrogen pairs but its shape crucially depends on the exact atomic configuration (Fig. 1). Calculation of formation energies for nitrogen pairs was shown to be favorable for the 1-4 (or 'para') configuration $[34,36]$. However, both postgrowth and direct doping methods have led experimentally to a prevalence of the 1-3 (or 'meta') configuration $[31,34,36]$, suggesting that kinetic factors might play an important role during formation of the defects. Notably, Lv et al. reported the CVD synthesis of samples with predominantly nitrogen pairs in the 1-3 ('meta') configu- ration, with $80 \%$ of the dopants' sites being of this type $[34]$.

Increase of the elastic tunneling channel at defects' sites

As previously mentioned, above a tip-sample bias threshold of about $58 \mathrm{mV}[45,46]$, the tunneling spectrum of graphene is dominated by inelastic tunneling channels $[44,45,47,49,50]$. In the presence of dopant, the situation changes dramatically. Lagoute et al. showed that, due to symmetry breaking, an elastic channel opens up leading to an enhancement of the conductance by a factor of up to 500 around the Fermi level [65]. This was evidenced by measuring at constant height $\mathrm{d} I / \mathrm{d} V$ spectra above graphene and above a graphitic nitrogen, as shown in Figs. 8a and 8b. The spectra in Fig. 8a were acquired with the same current and sample bias setpoint above the graphene and above the nitrogen dopant whereas the tip-sample distance was kept the same for the spectra in Fig. 8b (constant height mode), providing a reliable comparison. In this constant height condition, an up to 500 fold increase of the current is observed above nitrogen dopant at the Fermi level, suggesting great potential of N-doped graphene for electron injection in vertical heterostructures. This large increase of conductance corresponds to an increase in the voltage-dependent current decay length $\lambda$. As reported in Fig. 8c, $\lambda$ has a very weak voltage dependence above nitrogen, contrary to the situation above pristine graphene. It is approximately twice larger above nitrogen dopant than above pristine graphene, at the Fermi level. The spatial extent of the modification of $\lambda$ around nitrogen dopants can be visualized in Fig. 8d, where a spatial map for the value of $\lambda$ at $20 \mathrm{mV}$ is shown. Similar behavior has been recently reported for hydrogenated graphene [66].

As a result of the decay length being much larger above nitrogen than above pristine graphene, an important increase of the apparent height of the nitrogen atom is observed on STM images. Simulations of STM images of nitrogen dopants have yet only considered an elastic tunneling process. Consequently, although the main features (symmetry, position of the maxima) of the computed images reproduce well the experimental images, the computed apparent height of the dopant should be taken cautiously.

\section{Beyond simple substitution}

As illustrated in Fig. 1, several stable configurations of N-doping do not involve substitution but carbon vacancies. X-ray photoemission spectroscopy (XPS) is the most common tool to differentiate these various configurations even if the absolute binding energies for the three main N configurations (graphitic, pyridinic, and pyrrolic) vary significantly throughout the literature [5, 32, 67] and has been shown to depend on the nitrogen concentration 

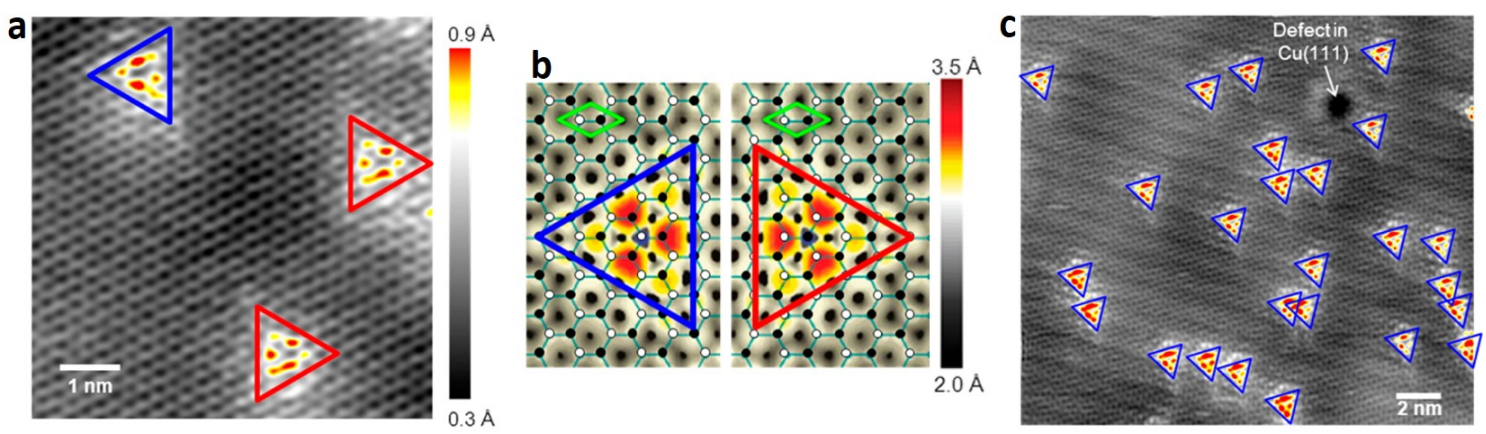

FiguRE 7. Sublattice segregation of nitrogen dopants observed by STM. (a) STM image $\left(7 \times 7 \mathrm{~nm}^{2}, 1 \mathrm{~V}, 1 \mathrm{nA}\right)$ of nitrogen-doped graphene on $\mathrm{Cu}(111)$ showing three dopants on two different sublattices. Bright colors in the STM image (red and yellow) correspond to carbon atoms surrounding nitrogen dopants, and red and blue triangles indicate dopants on different sublattices. (b) Simulated STM images of graphitic nitrogen dopants located on two different sublattices. Green diamonds indicate the unit cell of graphene, and black and white circles indicate the sublattices. Carbon atoms in the vicinity of the nitrogen dopants appear bright (red and yellow), forming triangles that appear as mirror images for the two sublattices (highlighted by red and blue triangles). (c) STM image $\left(20 \times 20 \mathrm{~nm}^{2}, 1 \mathrm{~V}, 1 \mathrm{nA}\right)$ of CVD (pyridine)-grown nitrogen-doped graphene, showing all dopants located on the same sublattice. From [63].
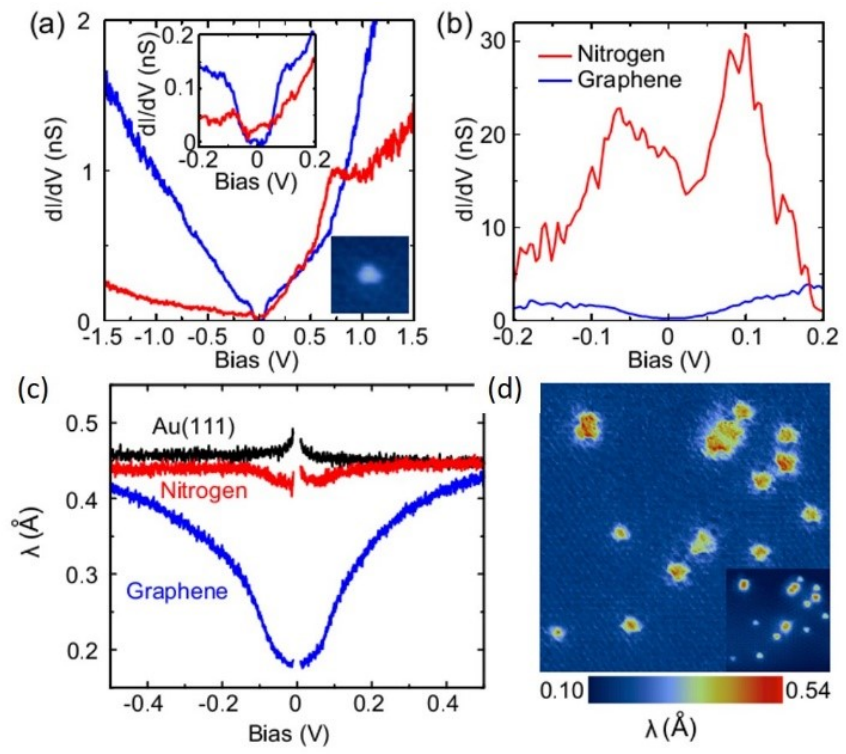

FIGURE 8. Increase of elastic tunneling above nitrogen dopant in graphene. (a) $\mathrm{d} I / \mathrm{d} V$ spectra measured above graphene far from the nitrogen atoms (blue) and above a nitrogen atom (red). The spectrum in inset is a zoom at low bias voltages of the spectra. The image in inset is a $2 \times 2 \mathrm{~nm}^{2}$ image of the nitrogen atom where the measurement was performed. (b) $\mathrm{d} I / \mathrm{d} V$ spectra around $E_{F}$ on carbon (blue) and nitrogen (red) sites measured in constant height mode conditions prior to the spectroscopy measurement (110 pA at $100 \mathrm{mV}$ above graphene carbon area). (c) Voltage dependent current decay length $\lambda$ measured on a reference $\mathrm{Au}(111)$ sample (black), $\mathrm{N}$-doped graphene carbon area (blue) and above a graphitic nitrogen atom in graphene (red). (d) $\lambda$ mapping at $20 \mathrm{mV}$ extracted from spectra measured at each point of the topographic image shown in the inset $\left(10 \times 10 \mathrm{~nm}^{2}, \mathrm{~V}=0.1 \mathrm{~V}\right.$, $\mathrm{I}=500 \mathrm{pA}$ ). From [65].
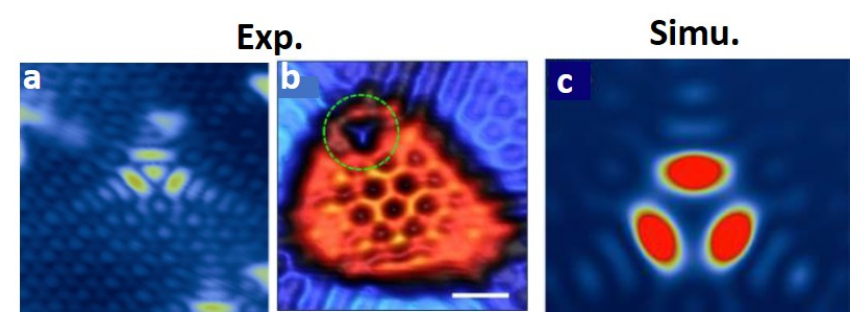

Figure 9. 3N-pyridine defect observed with STM. (a) STM image of $3 \mathrm{~N}$-pyridine in graphene on $\mathrm{SiC}(000 \overline{1})$. $3 \times 3 \mathrm{~nm}^{2} ; \mathrm{V}=-0.5 \mathrm{~V}$. (b) STM image of $3 \mathrm{~N}$-pyridine in graphene on $\mathrm{Ru}(0001)$. Scale bar is $0.5 \mathrm{~nm} ; \mathrm{V}=10 \mathrm{mV}, \mathrm{I}=1 \mathrm{nA}$. (c) DFT-based simulated STM image of a $3 \mathrm{~N}$-pyridine. (a) from [35]. (b) From [70]. (c) from [31].

[68]. However, the order of the binding energies is agreed upon : graphitic, pyrrolic, and pyridinic configurations have decreasing binding energies, respectively.

The most stable non-graphitic doping atomic configuration is the $3 \mathrm{~N}$-pyridine defect (Fig. 9a) [34, 69] with the same three fold symmetry (Fig. 9a) as the graphitic configuration. It has been observed by several groups [31, 35, 62, 70] (Fig. 9). As seen in STS spectra, the pyridinic defect differs from the graphitic configuration by its associated acceptor localized state [31, 67]. Some STM studies reported successful synthesis of samples with controlled proportion of this defect $[62,70]$. In particular, Ma et al. have demonstrated dominant pyridinic configuration of the dopants, by combining STM/STS and transport experiments [62]. 


\section{Molecular interaction}

The nitrogen doping modifies the interaction of graphene with molecular species. This effect has been exploited to perform catalysis, or electrochemical sensing with nitrogen-doped graphene [2, 71]. The electronic properties of molecules interacting with nitrogen sites has been investigated with scanning probe microscopy [61, 72-74]. STM experiments on free base tetraphenylporphyrin molecules $\left(\mathrm{H}_{2} \mathrm{TPP}\right)$ have shown that at nitrogen sites, the molecules undergo a downshift of their highest occupied molecular orbital (HOMO) and lowest unoccupied molecular orbital (LUMO) states, evidencing a local charge transfer at these sites (Fig. 10a and b) [72, 73]. Recently, it has been shown that in the case of electron acceptor tetracyanoquinodimethane (TCNQ) molecules the downshift of the LUMO state at nitrogen site is much larger than the shift observed with porphyrin molecules. DFT calculations revealed that a local charge transfer occurs at the nitrogen site, so that the molecule gains 0.33 electron, as compared to 0.20 electron at carbon sites (Fig. 10c and d) [74]. A combination of this effect with local gating allows to obtain a molecular layer with a mixed charge state. These experiments evidenced that the charge transfer and physicochemical properties of molecules on graphene are locally modified at nitrogen sites. In the case of magnetic molecules, STM combined with non contact AFM (nc-AFM) have revealed that the spin state of iron(II) phthalocyanine (FePc) is different at nitrogen and carbon sites allowing to control the spin state of the molecules by changing the molecular positioning on nitrogen-doped graphene [61] (Fig. 10e and f).

\section{B. Boron}

As a dopant, boron is complementary to nitrogen because of the expected hole-doping it was predicted to induce [41]. However, STM results on boron-doped graphene are scarce. Early STM imaging results were reported by Endo et al. on B-doped highly oriented pyrolytic graphite (HOPG). The first report of STM/STS experiments on B-doped graphene was by Zhao et al. [33] (Fig. 11), who produced B-doped graphene by CVD on copper foil, with $\mathrm{CH}_{4}, \mathrm{H}_{2}$, and $\mathrm{B}_{2} \mathrm{H}_{6}$ as precursors. The STM image (Fig. 11a) was very similar to the earlier simulation [41] with a large current on the top of the $\mathrm{B}$ atoms and of the three neighboring $\mathrm{C}$ atoms. As the same group did for nitrogen dopants, they established, through a combination of STM, DFT, and NEXAFS results, that the majority $(>80 \%)$ of the B dopants were incorporated in the graphitic form in the graphene lattice. They evidenced the expected hole-doping by STS (Fig. 11c) and evaluated at 0.5 the charge carrier (hole) given per dopant to the graphene lattice. They did not notice a sublattice asymmetry (Fig. 11b).

Subsequently, Lv et al. reported STM results on borondoped graphene grown with a triethylborane- $\left(\mathrm{C}_{6} \mathrm{H}_{15} \mathrm{~B}\right.$ - $)$ based bubbled-assisted CVD method. The most common doping site encountered in their sample was however attributed to a more complex configuration than the simple substitution [75]. Usachov et al. produced B-doped graphene with high B concentration (up to 19 at. \%) by CVD in $\mathrm{UHV}$, both on $\mathrm{Ni}(111)$ and on $\mathrm{Co}(0001)$ surfaces, using carborane $\left(\mathrm{C}_{2} \mathrm{~B}_{10} \mathrm{H}_{12}\right)$ and propylene $\left(\mathrm{C}_{3} \mathrm{H}_{6}\right)$ as precursors [76]. For low concentration (3.7 at. \%), they showed with STM that B-doped graphene on $\mathrm{Ni}(111)$ displayed great sublattice asymmetry in the dopants distribution. For higher concentration, when the structure becomes difficult to resolve with STM, the authors developed an XPS-based method to determine sublattice asymmetry and found that no asymmetry can be observed [76]. Note that the same group developed another XPS method, applicable to lower dopant concentration, to evidence sublattice segregation consisting in correlating the photondependence of the C1s and B1s peak energies [77]. Finally, we note that $\mathrm{Li}$ et al. have recently observed that adsorbed boron atoms induce localized magnetism, evidenced as spin-splitted peaks in the $\mathrm{d} I / \mathrm{d} V$ spectra in the vicinity of $E_{F}$ [78], similar to what was reported for adsorbed hydrogen [79].

\section{B-N codoping}

$\mathrm{B}$ and $\mathrm{N}$ co-doping of graphene can allow to tune the free charge carrier together with a modification of the chemical reactivity or catalytic behavior [80-82]. Early work in the context of co-doped carbon nanotubes demonstrated that BN domains in graphene is energetically more favorable than dopant dispersion [83]. Direct chemical conversion of graphene to hybrid layers has also been shown [84]. Ion sputtering deposition under $\mathrm{Ar} / \mathrm{CH}_{4}$ atmosphere allows control of the relative concentration but lead to a segregation of $\mathrm{C}$ and $\mathrm{BN}$ domains for most of the concentration [85]. Telychko et al. produced B-doped graphene by growing graphene on $\mathrm{SiC}$ under an $\mathrm{Si}$ flux produced by heating an heavily B-doped Si wafer and characterized it by STM/AFM [86] and NEXAFS [87]. The post-growth nitrogen doping technique could then be applied to their sample to produce B-N co-doped samples, subsequently characterized by STM as shown in Fig. 12 [86]. Schiros et al. also produced B-N co-doped graphene but by CVD on copper foil using a mixture of $\mathrm{CH}_{4}, \mathrm{H}_{2}$, and $\mathrm{B}_{2} \mathrm{H}_{6}$ and $\mathrm{NH}_{3}$ gases (Fig. 13) [88]. Besides nitrogen and boron in the graphitic configuration (arrows in Fig. 13), they observed more complex defects. By comparison to DFT-based STM simulations, supported by NEXAFS and XPS results, these defects were identified as involving boron and nitrogen atoms, in the configurations shown in Fig. 13. As the authors noticed, the presence of these complex B-N defects at a rate that exceeds what would be expected for random distribution is indicative of the difficulty in growing $h$-BNC materials with homogeneous carbon distribution (without $h$-BN and graphene domain segregation) and thus, tunable bandgap [84, 85, 89]. That 

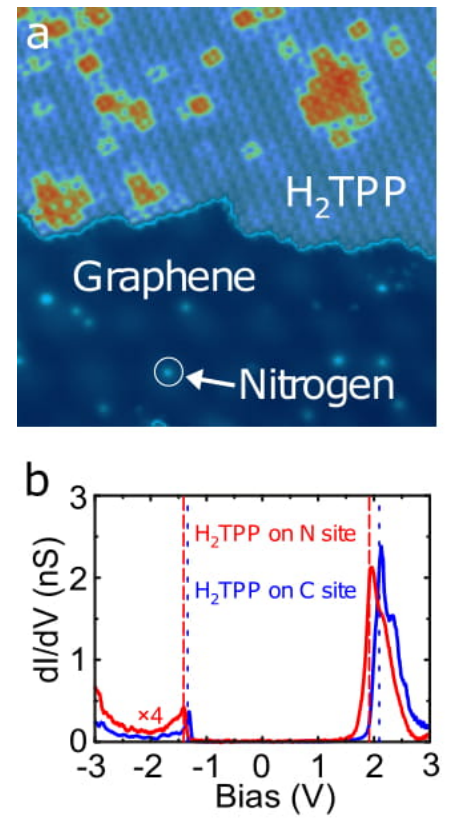
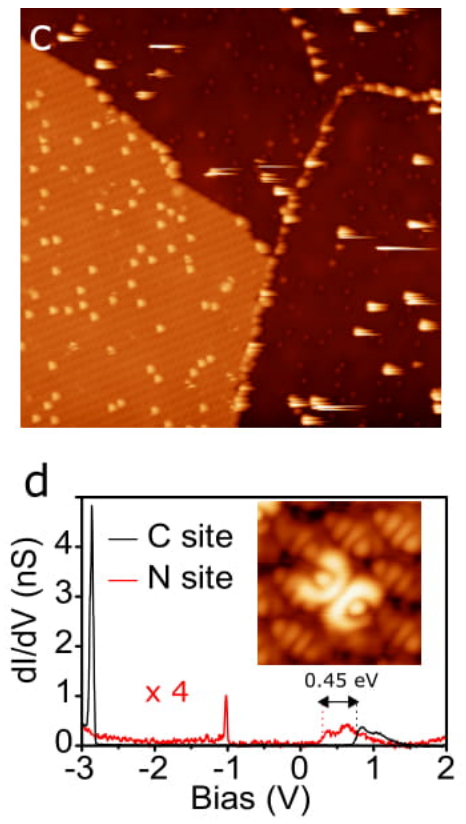
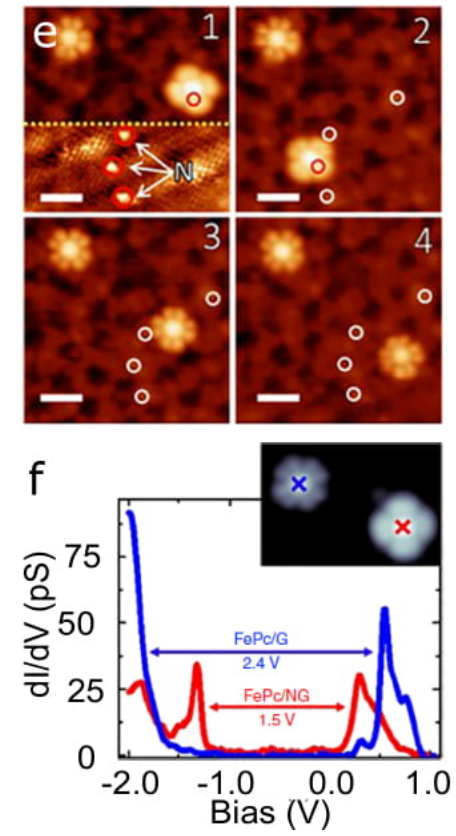

FIGURE 10. Interaction of organic molecules with nitrogen doped graphene. (a) STM image $(\mathrm{V}=2 \mathrm{~V})$ of $\mathrm{H}_{2} \mathrm{TPP}$ molecules on nitrogen-doped graphene on $\mathrm{SiC}(000 \overline{1})$. The molecules adsorbed on nitrogen sites appear brighter. (b) dI/dV spectra showing the shift to low energy of the spectrum of $\mathrm{H}_{2} \mathrm{TPP}$ molecules nitrogen (red) sites as compared to those on carbon sites (blue). The vertical dashed lines mark the energy position of the measured mean values of HOMO and LUMO states. (c) STM image of a TCNQ molecular island on nitrogen-doped graphene $\left(100 \times 100 \mathrm{~nm}^{2}, \mathrm{~V}=-1.5 \mathrm{~V}, \mathrm{I}=7 \mathrm{pA}\right)$. (d) $d I / d V$ spectra recorded above molecules adsorbed on a C-site and a N-site. The inset shows a topography image $\left(3 \times 3 \mathrm{~nm}{ }^{2}\right) \mathrm{revealing}$ the LUMO shape for a molecule adsorbed on a nitrogen site that appears in the occupied state region (-1.5 V). (e) STM images acquired after steps of controlled lateral manipulation of FePc on nitrogen-doped graphene. From the initial situation (1), where a FePc molecule is adsorbed on a nitrogen dopant, the molecule was moved to a different configuration on another dopant (2), then to a distance of $1.3 \mathrm{~nm}$ from any dopant (3), and finally to pristine graphene (4). The circles mark the positions of the dopants. The FePc molecule in the top left is located on pristine graphene and served as a reference. The scale bar is $2 \mathrm{~nm}$. (f) $\mathrm{dI} / \mathrm{dV}$ spectra acquired at the center of FePc on pristine graphene (blue) and on an nitrogen dopant (red). (a) and (b) from [72]. (c) and (d) from [74]. (e) and (f) From [61].

is supported by calculation of formation energies showing that there is a energy gain of about $2.35 \mathrm{eV}$ in forming a B-N defect (in a 1-2 configuration), rather than two isolated $\mathrm{B}$ and $\mathrm{N}$ defects [88].

\section{ANGLE-RESOLVED PHOTOEMISSION SPECTROSCOPY}

\section{A. Nitrogen}

The first ARPES characterization of N-doped graphene has been reported by Usachov et al. (Fig. 14) [90]. The sample was produced by CVD of s-triazine molecules $\left((\mathrm{HCN})_{3}\right)$ on a $\mathrm{Ni}(111)$ surface, followed by gold intercalation. They were able in this way to produce samples containing mostly graphitic nitrogen. For a nitrogen concentration of 0.48 at. $\%(0.40 \%$ and $0.08 \%$ in the graphitic and the pyridinic configuration, respectively, as measured by XPS ; $c f$. Fig. 14c), they measured a shift of the Dirac cone by $0.3 \mathrm{eV}$, corresponding to an average of 0.5 delocalized electron per dopant. They also inferred the creation of a band gap of $0.2 \mathrm{eV}$ from their ARPES data (Fig. 14a and 14b). One may note however that the existence of the band gap is not obvious from the reported experimental data, as the energy distribution curve at K (Fig. 14b) displays only a slight depression between the conduction and the valence bands (compared to e.g. the work of Balog et al. where a clear band gap creation can be deduced from ARPES data in the case of hydrogenated graphene [91]).

Subsequently, Koch et al. have reported ARPES data of $\mathrm{N}$-doped graphene also grown on $\mathrm{Ni}(111)$ by $\mathrm{CVD}$ in UHV but using pyridine $\left(\mathrm{C}_{5} \mathrm{H}_{5} \mathrm{~N}\right)$ as a precursor [92] They were however unable to convert the pyridinic nitrogen into its graphitic form and could therefore not evidence electronic doping. Velez-Fort et al. reported ARPES characterization of N-doped graphene produced by exposing $4 \mathrm{H}-\mathrm{SiC}(0001)$ to $\mathrm{N}_{2}$ during graphitization [93]. Despite a large amount (> 4 at. \%) of nitrogen atoms found to be in configurations other than the graphitic one, suggesting relatively high disorder, they evidenced the presence of graphitic nitrogen via XPS and the associated electronic doping via ARPES. Using also high nitrogen concentration $(\sim 8$ at. $\%)$ and a combi- 

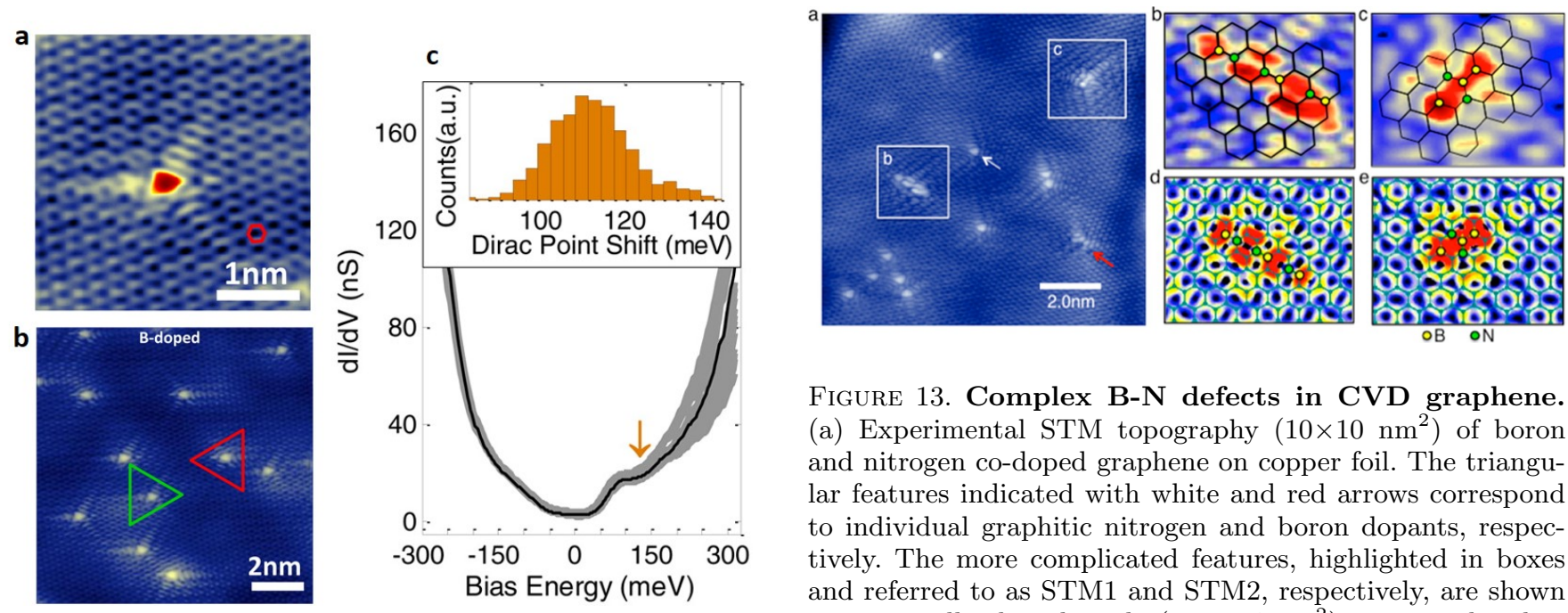

Figure 13. Complex B-N defects in CVD graphene. (a) Experimental STM topography $\left(10 \times 10 \mathrm{~nm}^{2}\right)$ of boron and nitrogen co-doped graphene on copper foil. The triangular features indicated with white and red arrows correspond to individual graphitic nitrogen and boron dopants, respectively. The more complicated features, highlighted in boxes and referred to as STM1 and STM2, respectively, are shown over a smaller length scale $\left(1.8 \times 1.4 \mathrm{~nm}^{2}\right)$ in more detail in (b) and (c), respectively, with a potential defect structure for (b) STM1 and (c) STM2 overlaid; boron and nitrogen dopant atoms are represented by yellow and green circles, respectively, surrounded by carbon host atoms at the vertices of the overlaid black honeycomb pattern. DFT-based simulated STM patterns for the BNC bond structures describing (d) STM1 and (e) STM2 produce excellent fits to experiment (ac) and allow atomic-level characterization of $\mathrm{B} / \mathrm{N}$ codopant structural motifs in single-layer graphene. From [88].

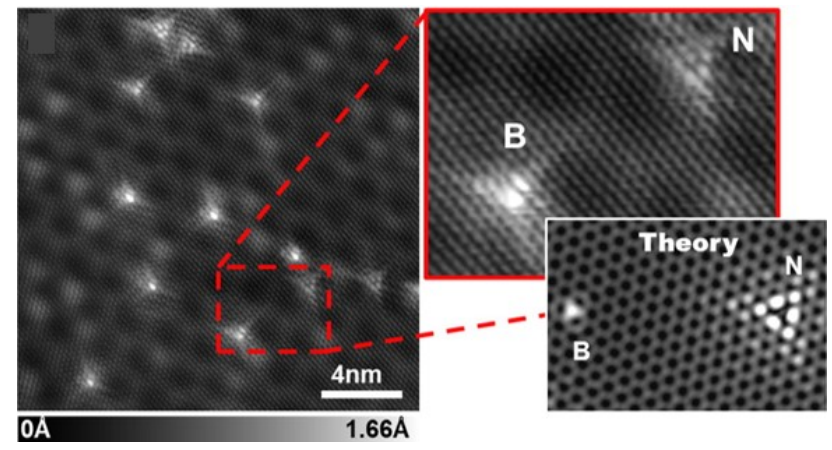

above. Also, STM allows a more reliable determination of the dopants' concentration (as compared to e.g. XPS) by counting directly the dopants on STM images. The combination of ARPES and STM on N-doped quasifreestanding graphene on $\mathrm{SiC}(000 \overline{1})$ [95] allowed revealing that the charge transferred per dopant depends on their concentration. For low N concentration $(<0.1$ at. \%), a significantly smaller transfer of electron per dopant was found than for larger concentration $(0.15$ vs. 0.4 for concentration up to 1 at. \%). This can be explained by the variation of the DOS of doped graphene which is in turn due to the variation of the position in energy of the resonance associated to the dopant.

The combination of ARPES and STS also permitted

FIGURE 12. B-N co-doping in graphene on $\mathrm{SiC}(0001)$ Constant-current STM image showing presence of N and B dopants in the graphene lattice $(\mathrm{V}=-0.7 \mathrm{mV} ; \mathrm{I}=0.15 \mathrm{nA}$; $20 \times 20 \mathrm{~nm}^{2}$ ). The inset shows a zoomed-in area with a detail of $\mathrm{N}$ and B single-atom dopants and a simulated STM image for comparison. From [86].

nation of ARPES and XPS measurements, Scardamaglia et al. have shown that pyridinic nitrogen obtained from room temperature plasma treatment of graphene grown on copper foil can be transformed to graphitic nitrogen by thermal heating at $500{ }^{\circ} \mathrm{C}$ [94].

Joucken et al. have combined STM and ARPES to tackle the charge transfer problem in N-doped graphene (Fig. 15) [68]. If the quality of the sample permits, ARPES allows indeed a more reliable determination of the electronic doping than STS, which suffers from the presence of inelastic tunneling channels, as discussed Joucken et al. to demonstrate the need for correcting the position in energy of features of interest in the STS spectra of graphene (such as $E_{D}$ ), thus providing further indication that the pseudogap around the Fermi level in STS spectra of graphene is due to the absence of phonon-mediated inelastic tunneling channels at low biases $[43,68]$. This was done by directly comparing the value for $E_{F}-E_{D}$ measured by ARPES with the one measured by STS. A value of $150 \mathrm{meV}$ was found by STS whereas a value of $85 \mathrm{meV}$ was found by ARPES. The value found by ARPES is assumed to be the real value so that the value found by STS has indeed to be renormalized by the phonon energy $(65 \mathrm{meV})$.

Finally, the effect of the substrate on the electronic doping efficiency of post-growth methods (ion bombardment) was investigated by Sforzini et al. [96]. They have 


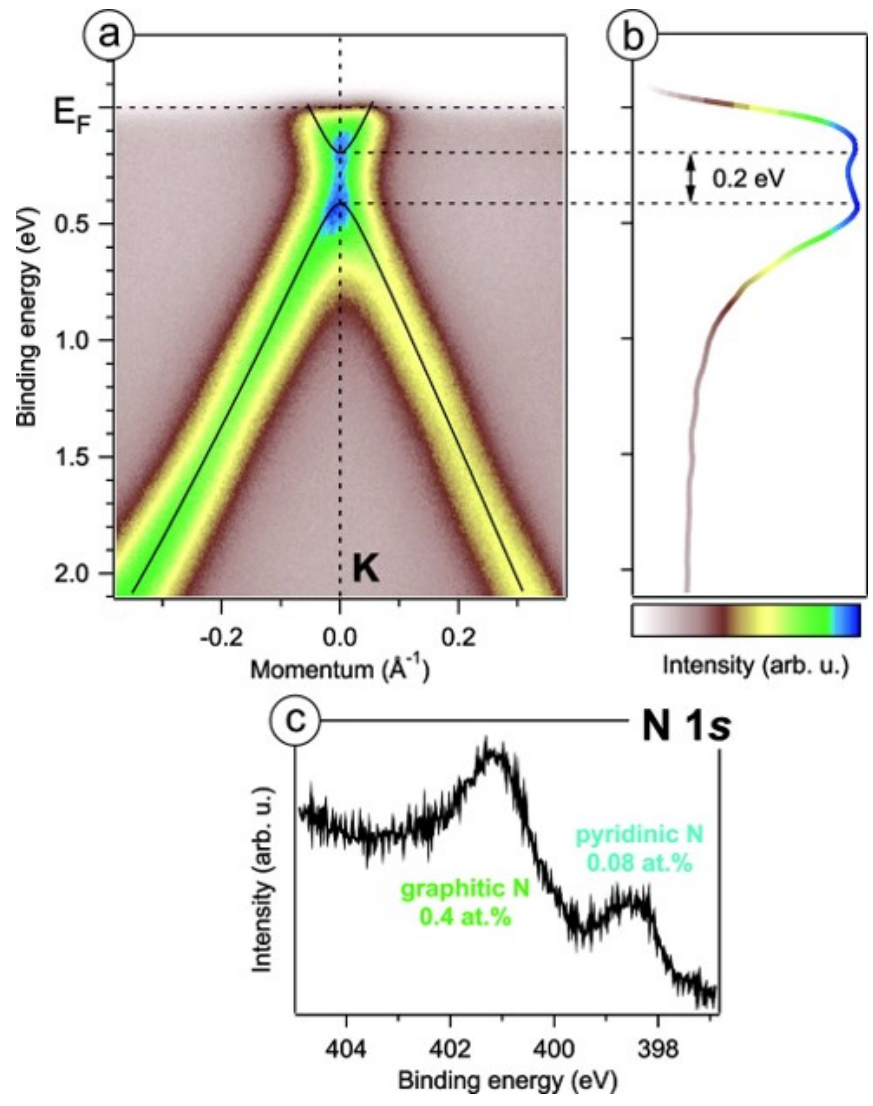

FIGURE 14. ARPES characterisation of N-doped graphene evidencing electron doping. (a) ARPES of Ngraphene/ $\mathrm{Au} / \mathrm{Ni}(111) / \mathrm{W}(110)$, measured at a photon energy of $35 \mathrm{eV}$, through the K-point, few degrees off the direction perpendicular to the $\Gamma K$ direction. (b) Energy distribution curve at the K-point. (c) XPS spectrum of the same sample around the N 1s core level. From [90].

used normal incidence x-ray scattering in conjunction with XPS and ARPES to show that the presence of the carbon buffer layer under the first graphene layer of graphene on $\mathrm{SiC}(0001)$ has a strong influence on the nitrogen doping efficiency [96]. More precisely, they showed that under the same doping conditions (same exposure time to the ions flux), the sample with carbon buffer layer contained twice as much graphitic nitrogen as the graphene sample without buffer layer while the electronic doping (shift of the $\pi$ band measured by ARPES) was comparable in both cases. The additional contribution to the doping was attributed to nitrogen atoms replacing hydrogen atoms at the $\mathrm{SiC} /$ graphene interface in the sample without buffer layer.

\section{B. Boron}

To our knowledge, Gebhardt et al. reported the first ARPES results on boron-doped graphene [97] (Fig. 16). The graphene was synthesized on Ni(111) by CVD using triethylborane as precursor. As evident from the ARPES data, the graphene $\pi$ band is strongly distorted around the K-point, due to interaction with the Ni $d$ bands [98]. Note also that the boron concentrations investigated in this study (0.045 to $0.28 \mathrm{ML}$ ) are considerably larger than what has been studied by STM $[33,86]$. As shown in Fig. 16, a clear upwards shift of the graphene $\pi$ band is observed upon B doping, corresponding to the expected hole-doping. However, strong coupling to the metallic substrate complicates the interpretation of the experimental data. Although the shift matches roughly the shift expected for B-doped freestanding graphene sheets, it is not attributed to the extra holes brought by the B dopants (which are mainly not in the graphitic form) but rather to a charge transfer from the substrate arising from an adsorption of the boron dopants on the fcchollow sites.

To the best of our knowledge, the only other ARPES characterization of B-doped graphene was reported by Usachov et al. [76], in a study already discussed in section IB. Although the graphene bands suffered strong hybridization with the substrate $(\mathrm{Ni}(111)$ and $\mathrm{Co}(0001))$, hole doping was evidenced by ARPES.

\section{MAGNETORESISTANCE}

\section{Electronic transport in graphene}

We start this section by discussing the basics of electron transport and McCann's theory of MR on graphene, which is the standard for analyzing MR results. Compared to other $2 \mathrm{D}$ metals, graphene is special because of the reduced backscattering due to the chiral nature of its charge carriers $[99,100]$. Depending on the impurity potential responsible for scattering, the magnetoresistance can be either negative (weak localization - WL) or positive (weak anti-localization - WAL). In pristine samples, one can expect the magnetic field to increase backscattering because of the additional phase in the path of electrons that would interfere destructively in the absence of magnetic field [39, 99, 100]. This equates to positive MR (WAL) and holds for intravalley, pseudospin-conserving scattering events. However, other effects (such as trigonal warping or intervalley scattering) should be taken into account for describing the magnetoresistance in real samples and it turns out that WL (negative MR) is generally observed in pristine graphene at very low magnetic field, followed by WAL at higher field [101-103], although, depending on the temperature and the carrier density, WAL can also dominate at very low field [104].

As indicated by early STM results [32, 105], atomically sharp scattering centers such as nitrogen or boron dopants are expected to induce phase-coherent intervalley scattering and their effects on the magnetotransport properties of graphene are most often discussed within the theory developed by McCann et al. [100]. Within that theory, if we ignore the trigonal warping correction, the 

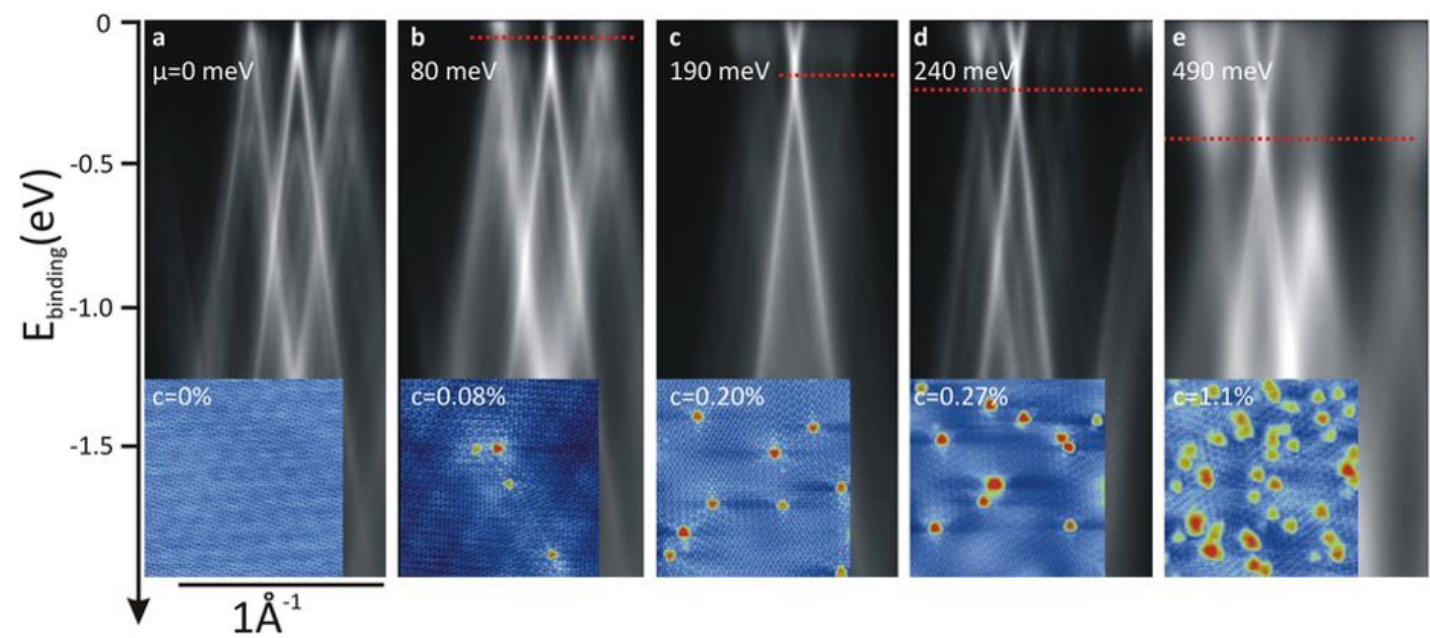

Figure 15. Combined ARPES and STM study of N-doped graphene. The shift of the Fermi level $\left(\mu=E_{F}-E_{D}\right)$ is given on each spectrum. Insets are typical $10 \times 10 \mathrm{~nm}^{2}$ STM images of the corresponding samples where the nitrogen dopants appear as red protrusions ; the nitrogen concentration (c) is given on each image. From [68].

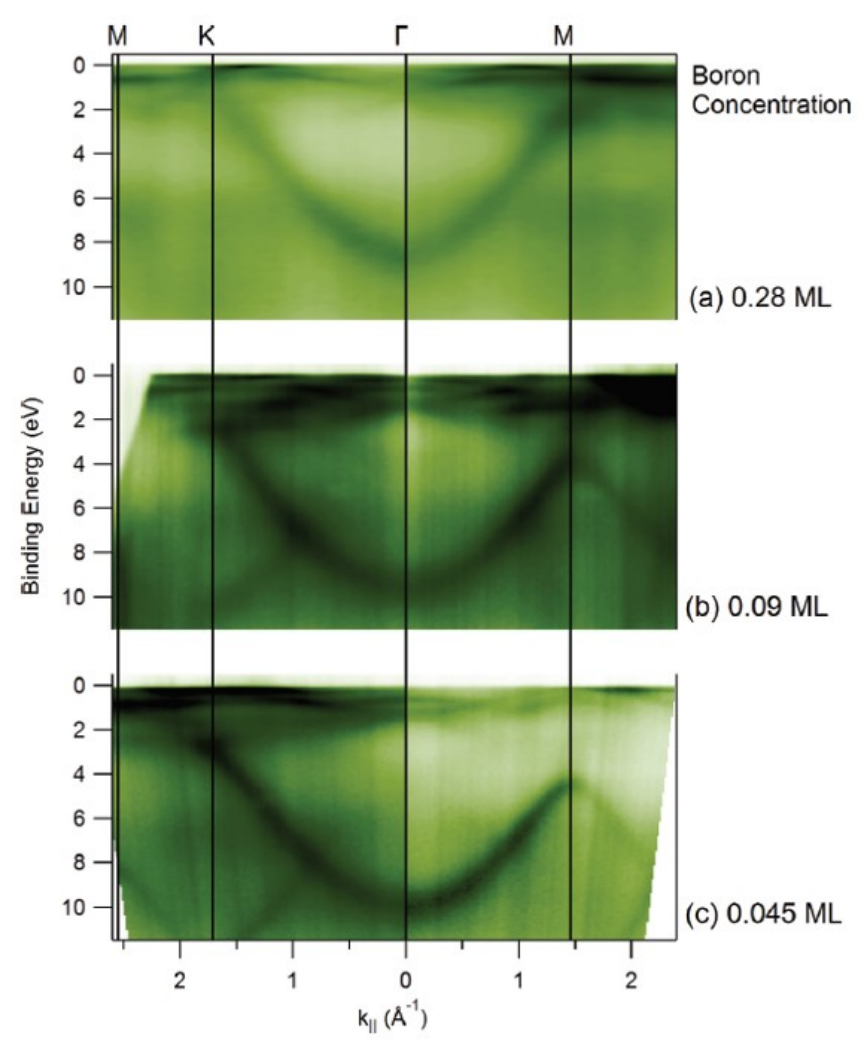

Figure 16. ARPES results on B-doped graphene on Ni(111). ARPES measurement along M-K- $-\mathrm{M}$ of borondoped graphene with boron concentrations of (a) $0.28 \mathrm{ML},(\mathrm{b})$ 0.09 ML, and (c) 0.045 ML. From [97]. variation in resistivity at low magnetic field reads

$$
\begin{aligned}
\Delta \rho(B)= & -\frac{\rho^{2} e^{2}}{\pi h} \times\left\{F\left(\frac{\tau_{B}^{-1}}{\tau_{\phi}^{-1}}\right)-F\left(\frac{\tau_{B}^{-1}}{\tau_{\phi}^{-1}+2 \tau_{\text {inter }}^{-1}}\right)\right. \\
& \left.-2 F\left(\frac{\tau_{B}^{-1}}{\tau_{\phi}^{-1}+\tau_{\text {inter }}^{-1}+\tau_{\text {intra }}^{-1}}\right)\right\},
\end{aligned}
$$

where $F(z)=\ln z+\psi(0.5+1 / z)$ ( $\psi$ is the digamma function), $\tau_{B}=\hbar /(4 e D B), \tau_{\phi}$ is the phase coherence time, and $\tau_{\text {inter }}$ and $\tau_{\text {intra }}$ are respectively the inter- and intravalley scattering times [106]. The diffusion constant $D$ is given by $D=v_{F}^{2} \tau$, where $\tau$ is the scattering time (about half of the transport time $\tau_{t r}$ for graphene $\left.[100,107]\right)$. The latter is generally extracted from gate-dependent conductivity measurements, following Boltzmann transport theory for Dirac fermions in graphene [108-111] : $\tau=\sigma_{D} h /\left(2 e^{2} v_{F} \sqrt{\pi n}\right)$, where the Drude conductivity $\sigma_{D}$ is the conductivity measured at high temperature (typically $100 \mathrm{~K}$ ), where localization effects are expected to vanish. The characteristic lengths are related to the characteristic times by $L_{\mu}=\sqrt{D \tau}$ and $L_{i}=\sqrt{D \tau_{i}}$, with $i=\phi$, inter or intra. $L_{\mu}$ is the carrier mean free path and $L_{\phi}$ is the coherence length i.e. the length over which the electron maintains its phase coherence. Finally, note that the first term in eq. (2) is responsible for localization while the other two terms are responsible for antilocalization.

As we discuss further below, a high level of doping, accompanied with structural defects, can lead to a transition from weak to strong (or Anderson) localization, when the coherence length $L_{\phi}$ becomes smaller than the localization length $L_{D}[112,113]$. The localization length can be extracted from temperature-dependent measurements assuming a variable-range hopping scenario, but it can also be estimated roughly as $L_{D}=L_{\mu} \exp \left(h \sigma_{D} / e^{2}\right)$ $[110,112,113]$. 
Magnetoresistance experiments on chemically-doped graphene

We now review the existing literature on MR of chemically-doped graphene, which is surprisingly rather limited. Before the first MR reports, $\mathrm{Li}$ et al. were the first to demonstrate electron doping through twoterminals transport measurements on nitrogen-doped graphene [114]. That was followed by other reports of electron-doping in N-doped graphene with two-terminals devices that showed reduced and asymmetric electron and hole mobilities associated with the incorporation of the nitrogen dopants [115-118]. The first report of MR measurements on chemically-doped graphene was by $\mathrm{Li}$ et al. (Fig. 17) [119]. Both pristine (Fig. 17a) and Ndoped (Fig. 17b) samples were grown by CVD on copper foil with methane as a precursor, adding $\mathrm{NH}_{3}$ for $\mathrm{N}$ doping. The samples were transferred to $\mathrm{SiO}_{2} / \mathrm{Si}$ for the transport experiments. Besides observing electron doping through Hall measurements, they extracted the characteristic scattering length scales for pristine and N-doped graphene (Fig. 17c) by fitting their MR results for pristine and N-doped graphene to a slightly modified McCann model adapted to high magnetic fields (Fig. 17c). As expected, they found a large increase for the intervalley scattering length in the $\mathrm{N}$-doped case, consistent with the more robust WL in the N-doped case compared to the pristine case (compare Fig. 17a and Fig. 17b).

Subsequently, Rein et al. reported MR measurements on $\mathrm{N}$-doped graphene also grown on copper foil by CVD with a $\mathrm{CH}_{4} / \mathrm{NH}_{3}$ mixture [120]. As shown in Fig. 18, they found drastically different MR response for pristine and $\mathrm{N}$-doped samples : both at $279 \mathrm{~K}$ and at $2.3 \mathrm{~K}$, undoped samples showed positive MR (except for very low field at low temperature) while $\mathrm{N}$-doped samples displayed negative MR in the whole B-field range investigated (up to $8 \mathrm{~T}$ ). The authors could not find a theory explaining the results at both temperatures. The linear dependence of the negative MR at large fields in the $\mathrm{N}$-doped samples was particularly difficult to account for. Focusing on the low field and low temperature results, they fit their data with the weak localization theory for general $2 \mathrm{D}$ metals [121] [122]. They obtained from these fittings coherence times $\tau_{\phi}$ 's and scattering times $\tau$ 's respectively of $0.33 \mathrm{ps}$ and $0.03 \mathrm{ps}$ for undoped graphene and $7.1 \mathrm{fs}$ and $6.3 \mathrm{fs}$ for $\mathrm{N}$-doped graphene. One can convert these time scales in length scales to obtain $L_{\phi}=70 \mathrm{~nm}$ and $L_{\mu}=21 \mathrm{~nm}$ for undoped graphene and $L_{\phi} \approx 5 \mathrm{~nm}$ and $L_{\mu} \approx 4 \mathrm{~nm}$ for $\mathrm{N}$-doped graphene. The value found for $L_{\phi}$ differs drastically from the value found for the same quantity by $\mathrm{Li}$ et al. (about $100 \mathrm{~nm}$ at that temperature) [119]. This indicates that the regimes examined in these two reports are very different. We note however that neither Li et al. nor Rein et al. provided estimations for the nitrogen concentration in their samples.

Wu et al. reported MR measurements for highly doped graphene ( $\sim 8$ at. \%) [123]. As visible on Fig. 19, the shape of the MR curve is drastically different from the
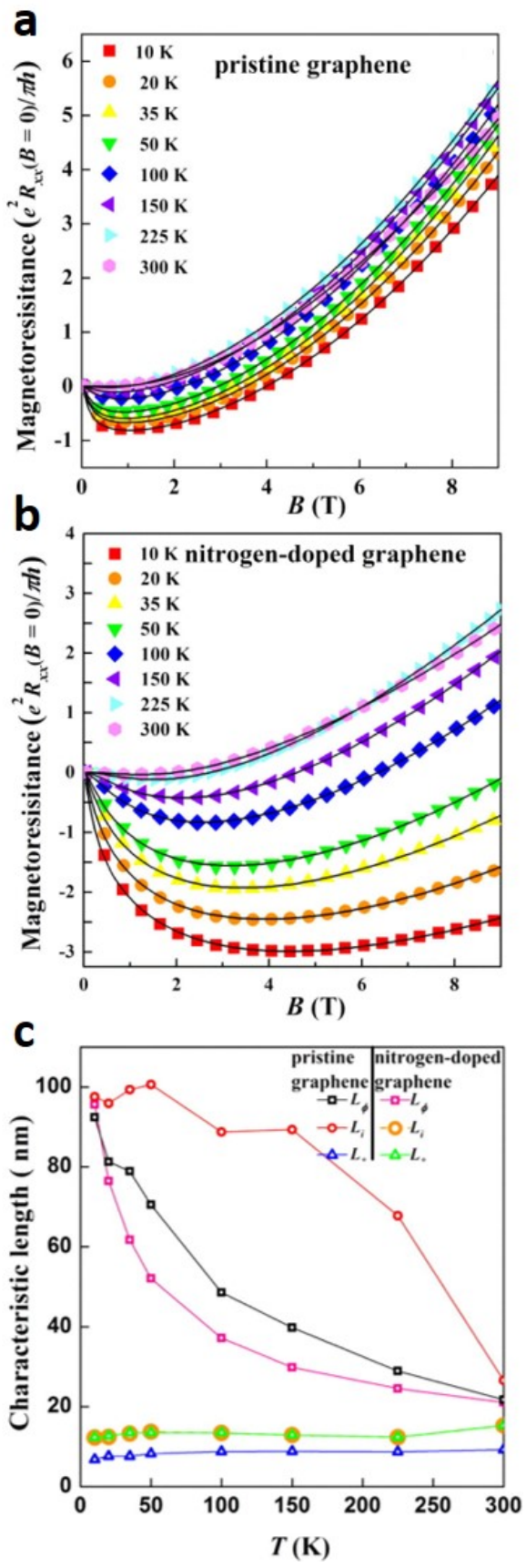

Figure 17. MR measurements on N-doped graphene. The temperature dependent magnetoresistance (normalized by $\left.e^{2} R_{x x}(B=0) / \pi h\right)$ for (a) pristine graphene and (b) nitrogen-doped graphene. (c) Comparison of characteristic lengths. From [119].

results discussed previously (Figs 17 and 18). ShubnikovDe Haas $(\mathrm{SdH})$ oscillations analysis showed that the amplitude of the $\mathrm{SdH}$ oscillations increases with nitrogen concentration and linearly decreases with increasing temperature, persisting up to a temperature of $150 \mathrm{~K}$. This dependence, at odds with the well-established LifshitzKosevich theory $[124,125]$, was tentatively explained by 

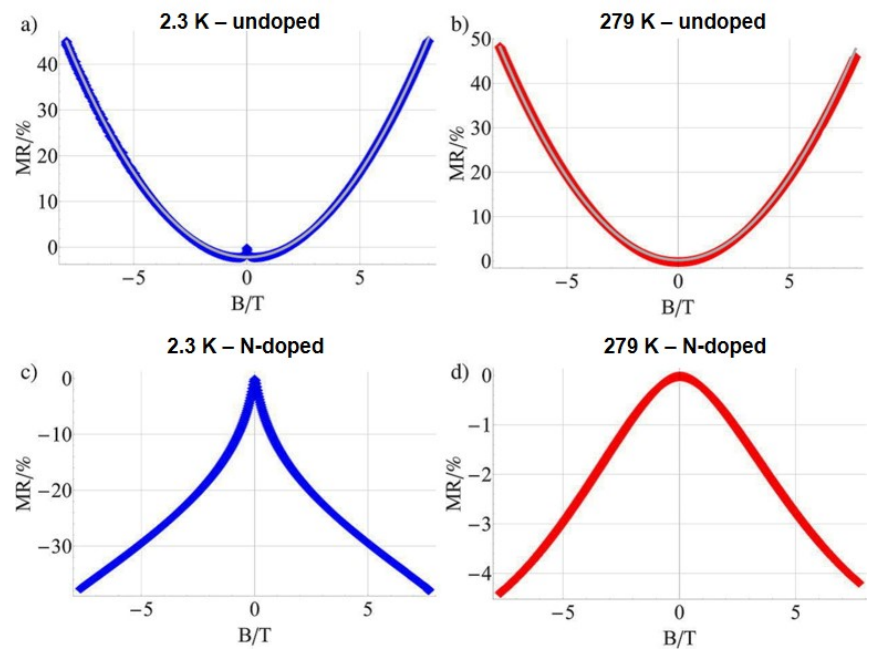

FIGURE 18. Magnetoresistance for doped and undoped graphene (magnetic fields applied perpendicular to the sample) for different temperatures. The upper line shows the undoped case for $2.3 \mathrm{~K}$ (a) and $279 \mathrm{~K}$ (b). At low temperature the weak localization peak is clearly visible at zero field. Graphs (c) and (d) show the same measurements for the doped case where the sign and the shape of the curves change compared to the pristine graphene. From [120].

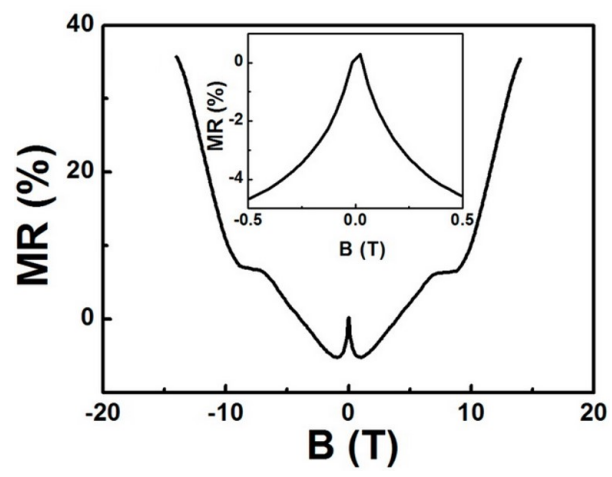

FiguRE 19. Magnetoresistance for nitrogen-doped in high magnetic field. MR measured at $10 \mathrm{~K}$ with field perpendicular to the graphene plane ( $\mathrm{N}$ concentration of $\sim 8$ at. \%). Inset : MR at low fields. From [123].

a dopants-induced bandgap which renormalizes the cyclotron mass and by a temperature dependence of the scattering time.

Willke et al. performed similar measurements on graphene samples grown on Si-terminated $\mathrm{SiC}$ and doped with both nitrogen and boron via ion implantation [56]. The originality of their work was to combine MR measurements with STM characterization on the same samples, so that they were able to evaluate the nitrogen and boron concentrations (slightly below 1 at. \% in each case), as well as the effectiveness of the doping (associated defects creation). The nitrogen doping was more effective than the boron doping, i.e. leading to fewer defects in the graphene lattice. The high magnetic field MR results (a)

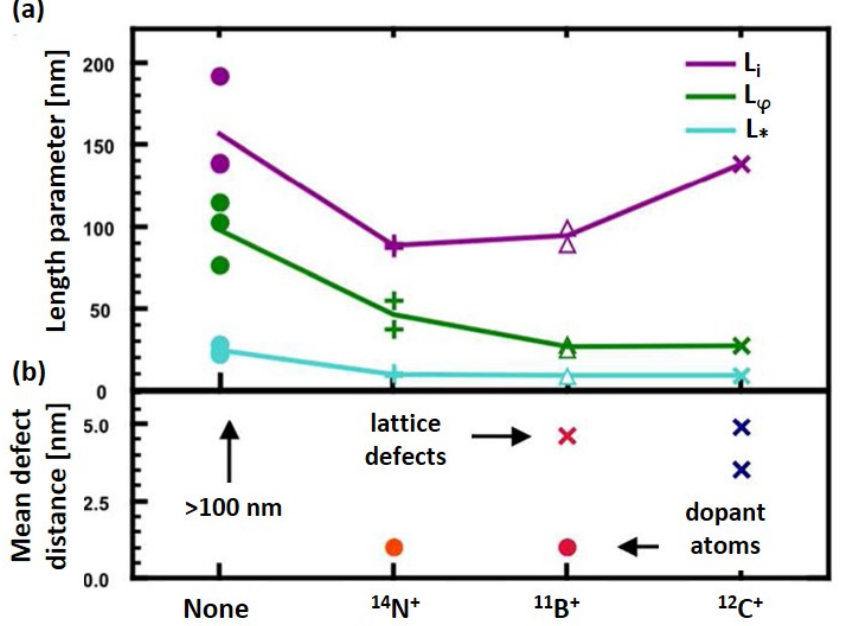

FIgURE 20. Coherence and scattering lengths for Nand B-doped graphene on SiC. (a) Intervalley scattering length $L_{i}$, phase coherence length $L_{\phi}$, and the combined scattering length $L_{*}$ derived from MR fits to McCann theory. (b) Mean defect distances derived from the STM topographies. While steps and interfaces are the only defects present for undoped samples ("None"), dopant atoms with a distance of approximately $1 \mathrm{~nm}$ can be found in nitrogen- and borondoped samples. Boron- and carbon-implanted samples show additional lattice defects with approximately $5 \mathrm{~nm}$ mean distance. Comparison to a purposely defective sample (labeled $\left." 13 \mathrm{C}^{+} "\right)$ is also provided. From [56].

they obtained are comparable to the results obtained by Rein et al. [120] : they observed a transition from positive to negative MR when doping their samples. However, the amplitude of the negative MR was significantly smaller for nitrogen-doped samples, indicating that the lattice defects might play an important role in the appearance of the negative MR at large field. Comparison to defective graphene (labeled " ${ }^{13} C^{+}$" in Fig. 20b) also supported this hypothesis. The fitting results of the low magnetic field data to McCann's theory led to the length parameters reported in Fig. 20, where the average distances between dopants/defects is also compared. The results for nitrogen- and boron-doped samples are comparable, with all lengths (coherence, inter-, and intravalley scattering lengths) divided by $\sim 2-3$ upon doping.

Using an ion implantation method for producing Ndoped graphene, Friedman et al. have investigated the effects of the $\mathrm{N}^{+}$ion energy on the transport properties of N-doped graphene through MR measurements [113] (cf. also ref. [59] for more details on the synthesis and further characterization). The graphene films were grown by CVD on copper foil, doped whilst on the copper foil and then transferred to $\mathrm{SiO}_{2} / \mathrm{Si}$ wafers for MR characterization. As opposed to the studies described so far in this review, they could tune the carrier density with the $\mathrm{Si}$ back-gate. For the ion energy that produces the most efficient doping ( $45 \mathrm{eV}$; efficient meaning here little lattice 
defects creation), they found negative MR for all doses and for all carrier densities (Fig. 21a-c). By assuming that the conductivity is determined only by $\tau_{\text {inter }}$ and $\tau_{\text {intra }}$, they fit their data to McCann's model with only the coherence time as a fitting parameter. They could then compare the coherence length to the localization length (estimated as $\left.L_{D}=L_{\mu} \exp \left(h \sigma_{D} / e^{2}\right)\right)$ to evaluate the localization regimes (weak or strong), as a function of carrier concentration and ion doses (Fig. 21d). The result is surprising since near the charge neutrality point (CNP), all doped samples show strong localization (an undoped witness sample showed $L_{\phi} / L_{D} \sim 1 \times 10^{-10}$ at the CNP) but amplitude of the localization increases steadily with decreasing nitrogen doses. The authors could not explain this puzzling behavior. They however further showed that for the same dose, increasing ion energy leads to larger MR, consistent with less efficient doping. This was supported by temperature-dependent conductivity measurements fitted by a superposition of a variable-range hopping and a thermal activation model.

Perhaps the most comprehensive study on the electronic transport properties of $\mathrm{N}$-doped graphene so far was by Li et al. [126] (Fig. 22). They grew N-doped graphene by CVD using a melamine pretreatment [127] and transferred it onto $\mathrm{SiO}_{2} / \mathrm{Si}$ for electronic transport characterization. Nitrogen concentrations (estimated by XPS measurements) of $2.0,1.8,1.0$, and 0.5 at. $\%$ were studied. Remarkably, mobility of $8,000 \mathrm{~cm}^{2} /(\mathrm{V} \cdot \mathrm{s})$ was reported for $\mathrm{N}$ concentration of 2 at. $\%$, indicating very high sample quality. This high sample quality allowed evidencing a clear electron-hole asymmetry in the intervalley scattering time through low magnetic field MR measurements (Fig. 22c), with a smaller intervalley scattering time on the electron side, and the strength of this asymmetry was found to be roughly proportional to the nitrogen concentration (Fig. 22d). They also found an electronhole asymmetry in the phase coherence length. The coherence length followed a linear/parabolic temperature dependence on the hole/electron side. Electron-hole asymmetry was also found at high magnetic field, where it was seen that the quantum Hall states were more robustly defined on the hole side than on the electron side. All these observations were tentatively explained by the fact that the nitrogen dopant, after providing electron to the graphene lattice, acts as a positively-charged scattering center with different effects on electrons and on holes : positive holes would be scattered off the nitrogen with smaller scattering angle [126]. Note that indications of such an electron-hole asymmetry in electronic transport was reported in earlier work [115, 118]. Finally, note that the same group has recently reported comprehensive results on nitrogen cluster doping [128]. Remarkably, compared to random doping, clustering allows higher mobility for similar electron doping. This is explained by the concentration of the scattering sources around the dopants' clusters [128].

\section{DISCUSSION AND PERSPECTIVES}

A lot of progress has been made in characterizing the electronics properties of chemically-doped graphene. In this last section, we will point towards what we believe would permit understanding the effect of the dopants on a deeper level.

We first note that the vast majority of the electronic properties characterization through STM/S, ARPES, or MR measurements have focused on nitrogen. Borondoped graphene has also been characterized, but to a much smaller extend and almost solely with STM/S. Other elements like aluminum [129, 130], phosphorus [131], sulfur [22], or transition metals [132-135] have also been envisioned or already tested for important applications such as hydrogen storage or oxygen reduction reactions. To our knowledge however, only Mo-[136] and recently Ni-doped [137] graphene have been characterized with STM, calling for further investigation of the electronic properties of graphene doped with these elements.

The determination of the fraction of the electron or hole provided by the dopant to the graphene lattice for graphitic nitrogen $(\sim 0.4[32,68])$ and boron $(\sim 0.5[33])$ dopants has been achieved, but only for a relatively narrow range of dopant concentration. For nitrogen, the interaction between dopants has been shown to modify the position of the resonant electronic state localized on the dopant [31]. Thus, for large concentration (>1 at. \%), the electronic doping per dopant is expected to decrease. Systematic data in this regime is however missing. Similarly, for low dopant concentration $(<0.1$ at. \%), experimental data and TB calculation suggests a reduction of electronic doping per dopant [68], but only a single experimental data point has been so far reported for that regime [68].

Spectroscopic measurements (STS or ARPES) of chemically-doped graphene on an insulating substrate are missing. Also, to date, no MR results have been reported for chemically-doped graphene on $h$-BN. Hexagonal boron nitride is however the substrate of choice for graphene-based electronics, in terms of electronic mobility, flatness, or substrate-induced electronic doping homogeneity [138-140]. MR experiments on this substrate would thus certainly bring deeper insights on the scattering mechanisms in doped samples. Subtle effects such as the influence of the dopants configuration or sublattice asymmetry could be studied on this substrate. Local tunneling spectroscopy experiments on an insulating substrate that allows gating would also permit studying the influence of the electronic doping on the resonant state associated with the dopant.

Density functional calculations suggest that, under certain configuration and concentration, nitrogen can induce magnetism in graphene $[141,142]$. Only recently mass magnetic susceptibility measurements have demonstrated the ferromagnetism induced by graphitic nitrogen dopant in graphene [143]. The ferromagnetism was triggered only for large nitrogen concentration ( $\sim 5$ at. \%) 
(a)

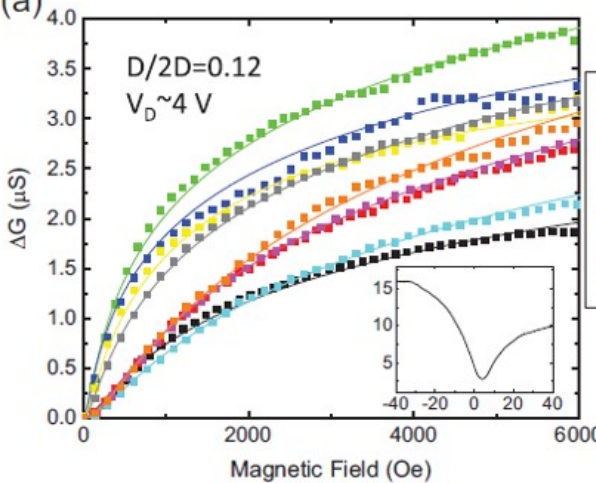

(c)

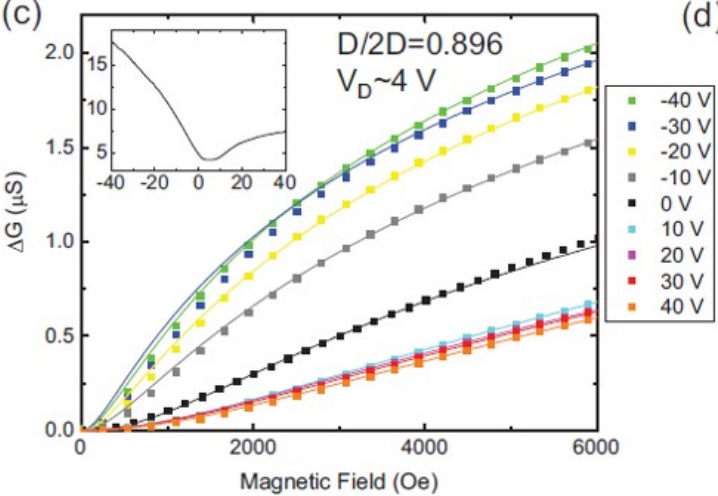

(b)

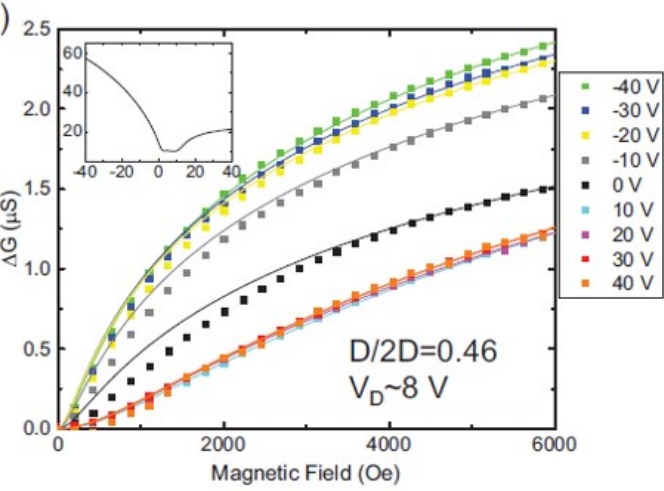

d)

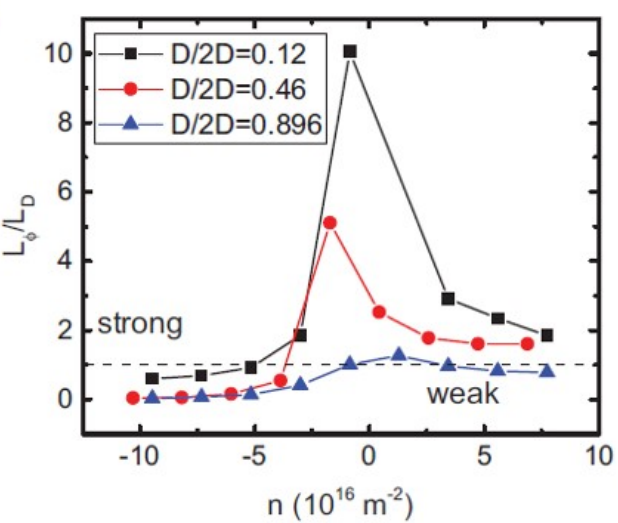

FIGURE 21. Gate-dependent localization analysis of $\mathbf{N}$-doped graphene. (a)-(c) Conductance vs. magnetic field at a variety of back-gate voltages for devices with $\mathrm{D} / 2 \mathrm{D}$ ratios of $0.12,0.46$, and 0.896 , respectively. The dotted lines show the data and the solid lines show the fits to the $2 \mathrm{D}$ weak localization model. The insets show the conductance $(\mathrm{y}$ axis $: \mu \mathrm{S})$ vs. back-gate voltage ( $\mathrm{x}$ axis : $\mathrm{V}$ ). Ion beam energy for these devices was $45 \mathrm{eV}$. (d) Ratio of fitting parameters extracted from the fits in (a)-(c) vs. carrier concentration for the three $\mathrm{D} / 2 \mathrm{D}$ ratios. Above the dotted line is strong localization and below the dotted line is weak localization. From [113].

and the Curie temperature was $\sim 70 \mathrm{~K}$. The authors performed DFT calculations which indicated that the 14 nitrogen pair configuration was particularly favorable for triggering magnetism in graphene. The same group also revealed ferromagnetism for sulfur-doped graphene [144]. Tunneling spectroscopy measurements could bring further evidence for these results and a detailed understanding of the magnetic sources at the atomic scale.

Finally, the interaction of chemically-doped graphene with molecules is a promising route to achieve advantageous functionalities. The local charge transfer at doping sites can change the nucleophilicity of adsorbed species that enhances their catalytic activity [145]. Single cobalt atoms at the surface of nitrogen-doped graphene have been shown to be efficient single atom catalyst [146]. Measures at the atomic scale of molecules on doped graphene are still scarce $[61,72,74]$ and demand more investigations on how the doping of graphene changes the dynamical, catalytic, electronic, and magnetic properties of adsorbed species.

\section{ACKNOWLEDGEMENTS}

The authors thank Robert Sporken and Philippe Lambin for their support and useful discussions.
[1] Y. Wang, Y. Shao, D. W. Matson, J. Li, and Y. Lin, Nitrogen-Doped Graphene and Its Application in Electrochemical Biosensing, ACS Nano 4, 1790 (2010).

[2] Z.-H. Sheng, X.-Q. Zheng, J.-Y. Xu, W.-J. Bao, F.-B. Wang, and X.-H. Xia, Electrochemical sensor based on nitrogen doped graphene : Simultaneous determination of ascorbic acid, dopamine and uric acid, Biosens. Bioe- lectron. 34, 125 (2012).

[3] L. Qu, Y. Liu, J.-B. Baek, and L. Dai, Nitrogen-Doped Graphene as Efficient Metal-Free Electrocatalyst for Oxygen Reduction in Fuel Cells, ACS Nano 4, 1321 (2010).

[4] D. Geng, Y. Chen, Y. Chen, Y. Li, R. Li, X. Sun, S. Ye, and S. Knights, High oxygen-reduction activity and du- 

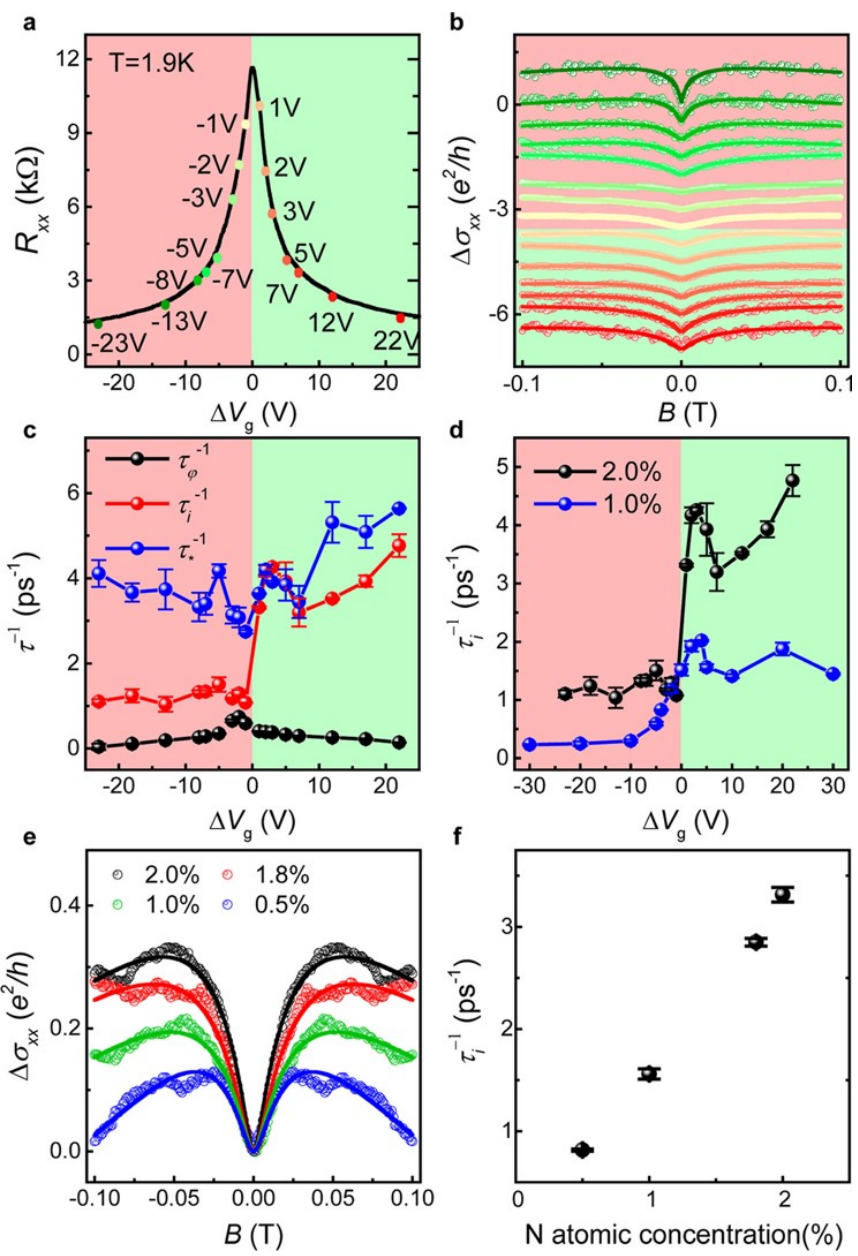

d
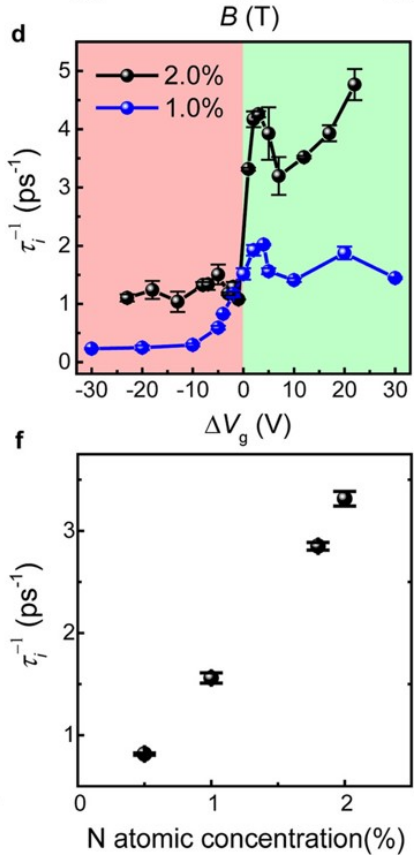

FIGURE 22. Electron-hole asymmetry in electronic transport in $\mathbf{N}$-doped graphene. (a) Shifted transfer curve of a $\mathrm{N}$-doped graphene sample with a 2 at. $\% \mathrm{~N}$ atomic concentration. In the originally measured transfer curve, the Dirac point is at $\mathrm{V}_{\mathrm{CNP}}=-16 \mathrm{~V}$. The regions shaded by different colors represent the regions of the transport with different types of charge carriers. The color-highlighted dots on the shifted transfer curve represent different gate voltages at which the measured magnetoconductivity is to be plotted. (b) Magnetoconductivity measured for the same sample as in (a) at selected back gate voltages as indicated by the colored dots in the shifted transfer curve shown in (a). The curve measured at $\Delta V_{g}=-23 \mathrm{~V}$ is placed on top and all the other measured curves are successively offset vertically by $-0.5 e^{2} / h$ for clarity. The solid lines show the best fits to the experimental data. (c) Scattering rates extracted for the same sample at different back gate voltages. (d) Intervalley scattering rates for the sample and a sample with 1.0 at. $\% \mathrm{~N}$ atomic concentration (Dirac point at $\mathrm{V}_{\mathrm{CNP}}=-4 \mathrm{~V}$ ) at different gate voltages. (e) Magnetoconductivity measured for samples with $\mathrm{N}$ atomic concentrations of $2.0,1.8,1.0$, and 0.5 at. $\%$, respectively on the electron transport side with the same carrier density of $n \sim 1 \times 10^{15} \mathrm{~m}^{-2}$. (f) Intervalley scattering rate extracted as a function of $\mathrm{N}$ atomic concentration from the measured magnetoconductivity curves shown in (e). From [126]. rability of nitrogen-doped graphene, Energy Environ. Sci. 4, 760 (2011).

[5] A. L. M. Reddy, A. Srivastava, S. R. Gowda, H. Gullapalli, M. Dubey, and P. M. Ajayan, Synthesis Of Nitrogen-Doped Graphene Films For Lithium Battery Application, ACS Nano 4, 6337 (2010).

[6] X. Zhou, L.-J. Wan, and Y.-G. Guo, Binding SnO2 Nanocrystals in Nitrogen-Doped Graphene Sheets as Anode Materials for Lithium-Ion Batteries, Adv. Mater. 25, 2152 (2013).

[7] H. Wang, C. Zhang, Z. Liu, L. Wang, P. Han, H. Xu, K. Zhang, S. Dong, J. Yao, and G. Cui, Nitrogen-doped graphene nanosheets with excellent lithium storage properties, J. Mater. Chem. 21, 5430 (2011).

[8] H. M. Jeong, J. W. Lee, W. H. Shin, Y. J. Choi, H. J. Shin, J. K. Kang, and J. W. Choi, Nitrogen-Doped Graphene for High-Performance Ultracapacitors and the Importance of Nitrogen-Doped Sites at Basal Planes, Nano Lett. 11, 2472 (2011).

[9] Z. Wen, X. Wang, S. Mao, Z. Bo, H. Kim, S. Cui, G. Lu, X. Feng, and J. Chen, Crumpled NitrogenDoped Graphene Nanosheets with Ultrahigh Pore Volume for High-Performance Supercapacitor, Adv. Mater. 24, 5610 (2012).

[10] L. Britnell, R. V. Gorbachev, R. Jalil, B. D. Belle, F. Schedin, A. Mishchenko, T. Georgiou, M. I. Katsnelson, L. Eaves, S. V. Morozov, N. M. R. Peres, J. Leist, A. K. Geim, K. S. Novoselov, and L. A. Ponomarenko, Field-Effect Tunneling Transistor Based on Vertical Graphene Heterostructures, Science (80-. ). 335, 947 (2012).

[11] J. R. Williams, L. DiCarlo, and C. M. Marcus, Quantum Hall Effect in a Gate-Controlled p-n Junction of Graphene, Science (80-. ). 10.1126/science.1144657 (2007).

[12] M. C. Lemme, F. H. L. Koppens, A. L. Falk, M. S. Rudner, H. Park, L. S. Levitov, and C. M. Marcus, GateActivated Photoresponse in a Graphene $\mathrm{p}-\mathrm{n}$ Junction, Nano Lett. 11, 4134 (2011).

[13] N. M. Gabor, J. C. W. Song, Q. Ma, N. L. Nair, T. Taychatanapat, K. Watanabe, T. Taniguchi, L. S. Levitov, and P. Jarillo-Herrero, Hot Carrier-Assisted Intrinsic Photoresponse in Graphene, Science (80-. ). 334, 648 (2011).

[14] M. Freitag, T. Low, and P. Avouris, Increased Responsivity of Suspended Graphene Photodetectors, Nano Lett. 13, 1644 (2013).

[15] B. Guo, L. Fang, B. Zhang, and J. R. Gong, Graphene Doping : A Review, Insciences J. 1, 80 (2011).

[16] H. Wang, T. Maiyalagan, and X. Wang, Review on Recent Progress in Nitrogen-Doped Graphene : Synthesis, Characterization, and Its Potential Applications, ACS Catal. 2, 781 (2012).

[17] X.-K. Kong, C.-L. Chen, and Q.-W. Chen, Doped graphene for metal-free catalysis, Chem. Soc. Rev. 43, 2841 (2014).

[18] L. K. Putri, W.-J. Ong, W. S. Chang, and S.-P. Chai, Heteroatom doped graphene in photocatalysis : A review, Appl. Surf. Sci. 358, 2 (2015).

[19] T. Susi, T. Pichler, and P. Ayala, X-ray photoelectron spectroscopy of graphitic carbon nanomaterials doped with heteroatoms, Beilstein J. Nanotechnol. 6, 177 (2015).

[20] S. Agnoli and M. Favaro, Doping graphene with boron : a review of synthesis methods, physicochemical charac- 
terization, and emerging applications, J. Mater. Chem. A 4, 5002 (2016).

[21] M. Kaur, M. Kaur, and V. K. Sharma, Nitrogen-doped graphene and graphene quantum dots : A review onsynthesis and applications in energy, sensors and environment (2018).

[22] Z. Yang, Z. Yao, G. Li, G. Fang, H. Nie, Z. Liu, X. Zhou, X. Chen, and S. Huang, Sulfur-Doped Graphene as an Efficient Metal-free Cathode Catalyst for Oxygen Reduction, ACS Nano 6, 205 (2012).

[23] S. Yang, L. Zhi, K. Tang, X. Feng, J. Maier, and K. Müllen, Efficient Synthesis of Heteroatom ( $\mathrm{N}$ or $\mathrm{S})-$ Doped Graphene Based on Ultrathin Graphene OxidePorous Silica Sheets for Oxygen Reduction Reactions, Adv. Funct. Mater. 22, 3634 (2012).

[24] Y. Li, J. Wang, X. Li, D. Geng, M. N. Banis, Y. Tang, D. Wang, R. Li, T.-K. Sham, and X. Sun, Discharge product morphology and increased charge performance of lithium-oxygen batteries with graphene nanosheet electrodes : the effect of sulphur doping, J. Mater. Chem. 22, 20170 (2012).

[25] P. Karthika, N. Rajalakshmi, and K. S. Dhathathreyan, Phosphorus-Doped Exfoliated Graphene for Supercapacitor Electrodes, J. Nanosci. Nanotechnol. 13, 1746 (2013).

[26] C. Zhang, N. Mahmood, H. Yin, F. Liu, and Y. Hou, Synthesis of Phosphorus-Doped Graphene and its Multifunctional Applications for Oxygen Reduction Reaction and Lithium Ion Batteries, Adv. Mater. 25, 4932 (2013).

[27] Y. Wen, B. Wang, C. Huang, L. Wang, and D. HulicovaJurcakova, Synthesis of Phosphorus-Doped Graphene and its Wide Potential Window in Aqueous Supercapacitors, Chem. - A Eur. J. 21, 80 (2015).

[28] F. Niu, L.-M. Tao, Y.-C. Deng, Q.-H. Wang, and W.G. Song, Phosphorus doped graphene nanosheets for room temperature NH3 sensing, New J. Chem. 38, 2269 (2014).

[29] B. Shen, J. Chen, X. Yan, and Q. Xue, Synthesis of fluorine-doped multi-layered graphene sheets by arcdischarge, RSC Adv. 2, 6761 (2012).

[30] K. Kakaei and A. Balavandi, Hierarchically porous fluorine-doped graphene nanosheets as efficient metalfree electrocatalyst for oxygen reduction in gas diffusion electrode, J. Colloid Interface Sci. 490, 819 (2017).

[31] Y. Tison, J. Lagoute, V. Repain, C. Chacon, Y. Girard, S. Rousset, F. Joucken, D. Sharma, L. Henrard, H. Amara, A. Ghedjatti, and F. Ducastelle, Electronic Interaction between Nitrogen Atoms in Doped Graphene, ACS Nano 9, 670 (2015).

[32] L. Zhao, R. He, K. T. Rim, T. Schiros, K. S. Kim, H. Zhou, C. Gutiérrez, S. P. Chockalingam, C. J. Arguello, L. Pálová, D. Nordlund, M. S. Hybertsen, D. R. Reichman, T. F. Heinz, P. Kim, A. Pinczuk, G. W. Flynn, and A. N. Pasupathy, Visualizing Individual Nitrogen Dopants in Monolayer Graphene, Science (80-. ). 333, 999 (2011).

[33] L. Zhao, M. Levendorf, S. Goncher, T. Schiros, L. Pálová, A. Zabet-Khosousi, K. T. Rim, C. Gutiérrez, D. Nordlund, C. Jaye, M. Hybertsen, D. Reichman, G. W. Flynn, J. Park, and A. N. Pasupathy, Local Atomic and Electronic Structure of Boron Chemical Doping in Monolayer Graphene, Nano Lett. 13, 4659 (2013).
[34] R. Lv, Q. Li, A. R. Botello-Méndez, T. Hayashi, B. Wang, A. Berkdemir, Q. Hao, A. L. Elías, R. CruzSilva, H. R. Gutiérrez, Y. A. Kim, H. Muramatsu, J. Zhu, M. Endo, H. Terrones, J.-C. Charlier, M. Pan, and M. Terrones, Nitrogen-doped graphene : beyond single substitution and enhanced molecular sensing, Sci. Rep. 2, 586 (2012).

[35] F. Joucken, Y. Tison, J. Lagoute, J. Dumont, D. Cabosart, B. Zheng, V. Repain, C. Chacon, Y. Girard, A. R. Botello-Méndez, S. Rousset, R. Sporken, J. C. Charlier, and L. Henrard, Localized state and charge transfer in nitrogen-doped graphene, Phys. Rev. B - Condens. Matter Mater. Phys. 85, 161408 (2012), arXiv :1204.3560.

[36] M. Telychko, P. Mutombo, M. Ondráček, P. Hapala, F. C. Bocquet, J. Kolorenč, M. Vondráček, P. Jelínek, and M. Švec, Achieving High-Quality Single-Atom Nitrogen Doping of Graphene/SiC(0001) by Ion Implantation and Subsequent Thermal Stabilization, ACS Nano 8, 7318 (2014).

[37] M. S. Bittencourt, C. Struzzi, S. Osella, N. Reckinger, J.-F. Colomer, L. Petaccia, R. Snyders, D. Beljonne, and Carla, Tuning nitrogen species to control the charge carrier concentration in highly doped graphene, 2D Mater. 3, 11001 (2016).

[38] A. J. Marsden, P. Brommer, J. J. Mudd, M. A. Dyson, R. Cook, M. Asensio, J. Avila, A. Levy, J. Sloan, D. Quigley, G. R. Bell, and N. R. Wilson, Effect of oxygen and nitrogen functionalization on the physical and electronic structure of graphene, Nano Res. 8, 2620 (2015).

[39] A. H. Castro Neto, F. Guinea, N. M. R. Peres, K. S. Novoselov, and A. K. Geim, The electronic properties of graphene, Rev. Mod. Phys. 81, 109 (2009).

[40] K. S. Novoselov, A. K. Geim, S. V. Morozov, D. Jiang, Y. Zhang, S. V. Dubonos, I. V. Grigorieva, and A. A. Firsov, Electric Field Effect in Atomically Thin Carbon Films, Science (80-. ). 306, 666 (2004).

[41] B. Zheng, P. Hermet, and L. Henrard, Scanning Tunneling Microscopy Simulations of Nitrogen- and BoronDoped Graphene and Single-Walled Carbon Nanotubes, ACS Nano 4, 4165 (2010).

[42] P. Lambin, H. Amara, F. Ducastelle, and L. Henrard, Long-range interactions between substitutional nitrogen dopants in graphene : Electronic properties calculations, Phys. Rev. B 86, 45448 (2012).

[43] Y. Zhang, V. W. Brar, F. Wang, C. Girit, Y. Yayon, M. Panlasigui, A. Zettl, and M. F. Crommie, Giant phonon-induced conductance in scanning tunnelling spectroscopy of gate-tunable graphene, Nat. Phys. 4, 627 (2008).

[44] F. D. Natterer, Y. Zhao, J. Wyrick, Y. H. Chan, W. Y. Ruan, M. Y. Chou, K. Watanabe, T. Taniguchi, N. B. Zhitenev, and J. A. Stroscio, Strong Asymmetric Charge Carrier Dependence in Inelastic Electron Tunneling Spectroscopy of Graphene Phonons, Phys. Rev. Lett. 114, 245502 (2015).

[45] N. Néel, C. Steinke, T. O. Wehling, and J. Kröger, Inelastic electron tunneling into graphene nanostructures on a metal surface, Phys. Rev. B 95, 161410 (2017).

[46] Z. Li, S. Li, H.-Y. T. Chen, N. Gao, K. Schouteden, X. Qiang, J. Zhao, S. Brems, C. Huyghebaert, and C. Van Haesendonck, Strongly Hole-Doped and Highly Decoupled Graphene on Platinum by Water Intercalation, J. Phys. Chem. Lett. 10, 3998 (2019). 
[47] M. L. N. Palsgaard, N. P. Andersen, and M. Brandbyge, Unravelling the role of inelastic tunneling into pristine and defected graphene, Phys. Rev. B 91, 121403 (2015).

[48] D. Deng, X. Pan, L. Yu, Y. Cui, Y. Jiang, J. Qi, W.-X. Li, Q. Fu, X. Ma, Q. Xue, G. Sun, and X. Bao, Toward N-Doped Graphene via Solvothermal Synthesis, Chem. Mater. 23, 1188 (2011).

[49] Y. Zhang, T.-T. Tang, C. Girit, Z. Hao, M. C. Martin, A. Zettl, M. F. Crommie, Y. R. Shen, and F. Wang, Direct observation of a widely tunable bandgap in bilayer graphene, Nature 459, 820 (2009).

[50] V. W. Brar, S. Wickenburg, M. Panlasigui, C.-H. Park, T. O. Wehling, Y. Zhang, R. Decker, Ç. Girit, A. V. Balatsky, S. G. Louie, A. Zettl, and M. F. Crommie, Observation of Carrier-Density-Dependent Many-Body Effects in Graphene via Tunneling Spectroscopy, Phys. Rev. Lett. 104, 36805 (2010).

[51] G. Mándi and K. Palotás, Chen's derivative rule revisited : Role of tip-orbital interference in STM, Phys. Rev. B - Condens. Matter Mater. Phys. 91, 165406 (2015), arXiv :1503.09189.

[52] N. J. van der Heijden, D. Smith, G. Calogero, R. S. Koster, D. Vanmaekelbergh, M. A. van Huis, and I. Swart, Recognizing nitrogen dopant atoms in graphene using atomic force microscopy, Phys. Rev. B 93, 245430 (2016).

[53] S. H. Rhim, Y. Qi, Y. Liu, M. Weinert, and L. Li, Formation of nitrogen-vacancy complexes during plasmaassisted nitrogen doping of epitaxial graphene on SiC(0001), Appl. Phys. Lett. 100, 233119 (2012).

[54] Z.-j. Wang, M. Wei, L. Jin, Y. Ning, L. Yu, Q. Fu, and X. Bao, Simultaneous N-intercalation and N-doping of epitaxial graphene on $6 \mathrm{H}-\mathrm{SiC}(0001)$ through thermal reactions with ammonia, Nano Res. 6, 399 (2013).

[55] D. Wei, L. Peng, M. Li, H. Mao, T. Niu, C. Han, W. Chen, and A. T. S. Wee, Low temperature critical growth of high quality nitrogen doped graphene on dielectrics by plasma-enhanced chemical vapor deposition, ACS Nano 9, 164 (2015).

[56] P. Willke, J. A. Amani, A. Sinterhauf, S. Thakur, T. Kotzott, T. Druga, S. Weikert, K. Maiti, H. Hofsäss, and M. Wenderoth, Doping of Graphene by Low-Energy Ion Beam Implantation : Structural, Electronic, and Transport Properties, Nano Lett. 15, 5110 (2015).

[57] A. Martín-Recio, C. Romero-Muñiz, P. Pou, R. Pérez, and J. M. Gómez-Rodríguez, Purely substitutional nitrogen on graphene/Pt(111) unveiled by STM and first principles calculations, Nanoscale 8, 17686 (2016).

[58] J. Zhang, C. Zhao, N. Liu, H. Zhang, J. Liu, Y. Q. Fu, B. Guo, Z. Wang, S. Lei, and P. A. Hu, Tunable electronic properties of graphene through controlling bonding configurations of doped nitrogen atoms, Sci. Rep. 6, 28330 (2016), arXiv :0511711v1 [arXiv :cond-mat].

[59] C. D. Cress, S. W. Schmucker, A. L. Friedman, P. Dev, J. C. Culbertson, J. W. Lyding, and J. T. Robinson, Nitrogen-Doped Graphene and Twisted Bilayer Graphene via Hyperthermal Ion Implantation with Depth Control, ACS Nano 10, 3714 (2016).

[60] A. Martín-Recio, C. Romero-Muñiz, P. Pou, R. Pérez, and J. M. Gómez-Rodríguez, Combining nitrogen substitutional defects and oxygen intercalation to control the graphene corrugation and doping level, Carbon N. Y. 130, 362 (2018).
[61] B. de la Torre, M. Švec, P. Hapala, J. Redondo, O. Krejčí, R. Lo, D. Manna, A. Sarmah, D. Nachtigallová, J. Tuček, P. Błoński, M. Otyepka, R. Zbořil, P. Hobza, and P. Jelínek, Non-covalent control of spinstate in metal-organic complex by positioning on $\mathrm{N}$ doped graphene, Nat. Commun. 9, 2831 (2018).

[62] C. Ma, Q. Liao, H. Sun, S. Lei, Y. Zheng, R. Yin, A. Zhao, Q. Li, and B. Wang, Tuning the Doping Types in Graphene Sheets by N Monoelement, Nano Lett. 18, 386 (2018).

[63] A. Zabet-Khosousi, L. Zhao, L. Pálová, M. S. Hybertsen, D. R. Reichman, A. N. Pasupathy, and G. W. Flynn, Segregation of sublattice domains in nitrogendoped graphene, J. Am. Chem. Soc. 136, 1391 (2014).

[64] J. Neilson, H. Chinkezian, H. Phirke, A. Osei-Twumasi, Y. Li, C. Chichiri, J. Cho, K. Palotas, L. Gan, S. J. Garrett, K. C. Lau, and L. Gao, Nitrogen-Doped Graphene on Copper : Edge-Guided Doping Process and Doping-Induced Variation of Local Work Function, J. Phys. Chem. C 10.1021/acs.jpcc.8b11261 (2019).

[65] J. Lagoute, F. Joucken, V. Repain, Y. Tison, C. Chacon, A. Bellec, Y. Girard, R. Sporken, E. H. Conrad, F. Ducastelle, M. Palsgaard, N. P. Andersen, M. Brandbyge, and S. Rousset, Giant tunnel-electron injection in nitrogen-doped graphene, Phys. Rev. B 91, 125442 (2015).

[66] D. Wong, Y. Wang, W. Jin, H.-Z. Tsai, A. Bostwick, E. Rotenberg, R. K. Kawakami, A. Zettl, A. A. Mostofi, J. Lischner, and M. F. Crommie, Microscopy of hydrogen and hydrogen-vacancy defect structures on graphene devices, Phys. Rev. B 98, 155436 (2018).

[67] T. Schiros, D. Nordlund, L. Pálová, D. Prezzi, L. Zhao, K. S. Kim, U. Wurstbauer, C. Gutiérrez, D. Delongchamp, C. Jaye, D. Fischer, H. Ogasawara, L. G. M. Pettersson, D. R. Reichman, P. Kim, M. S. Hybertsen, and A. N. Pasupathy, Connecting dopant bond type with electronic structure in n-doped graphene, Nano Lett. 12, 4025 (2012).

[68] F. Joucken, Y. Tison, P. Le Fèvre, A. Tejeda, A. TalebIbrahimi, E. Conrad, V. Repain, C. Chacon, A. Bellec, Y. Girard, S. Rousset, J. Ghijsen, R. Sporken, H. Amara, F. Ducastelle, and J. Lagoute, Charge transfer and electronic doping in nitrogen-doped graphene, Sci. Rep. 5, 14564 (2015), arXiv :arXiv :1011.1669v3.

[69] S. Kattel, P. Atanassov, and B. Kiefer, Stability, electronic and magnetic properties of in-plane defects in graphene : A first-principles study, J. Phys. Chem. C 116, 8161 (2012).

[70] X. Fei, J. Neilson, Y. Li, V. Lopez, S. J. Garrett, L. Gan, H.-J. Gao, and L. Gao, Controlled Synthesis of Nitrogen-Doped Graphene on Ruthenium from Azafullerene, Nano Lett. 10.1021/acs.nanolett.7b00038 (2017).

[71] X. Feng, Y. Zhang, J. Zhou, Y. Li, S. Chen, L. Zhang, Y. Ma, L. Wang, and X. Yan, Three-dimensional nitrogen-doped graphene as an ultrasensitive electrochemical sensor for the detection of dopamine, Nanoscale 7, 2427 (2015).

[72] V. D. Pham, J. Lagoute, O. Mouhoub, F. Joucken, V. Repain, C. Chacon, A. Bellec, Y. Girard, and S. Rousset, Electronic interaction between nitrogendoped graphene and porphyrin molecules, ACS Nano 8, 9403 (2014).

[73] V. D. Pham, F. Joucken, V. Repain, C. Chacon, A. Bellec, Y. Girard, S. Rousset, R. Sporken, M. C. Dos San- 
tos, and J. Lagoute, Molecular adsorbates as probes of the local properties of doped graphene, Sci. Rep. 6, 24796 (2016).

[74] V. D. Pham, S. Ghosh, F. Joucken, M. PelaezFernandez, V. Repain, C. Chacon, A. Bellec, Y. Girard, R. Sporken, S. Rousset, Y. J. Dappe, S. Narasimhan, and J. Lagoute, Selective control of molecule charge state on graphene using tip-induced electric field and nitrogen doping, npj 2D Mater. Appl. 3, 5 (2019).

[75] R. Lv, G. Chen, Q. Li, A. McCreary, A. Botello-Méndez, S. V. Morozov, L. Liang, X. Declerck, N. Perea-López, D. A. Cullen, S. Feng, A. L. Elías, R. Cruz-Silva, K. Fujisawa, M. Endo, F. Kang, J.-C. Charlier, V. Meunier, M. Pan, A. R. Harutyunyan, K. S. Novoselov, and M. Terrones, Ultrasensitive gas detection of largearea boron-doped graphene, Proc. Natl. Acad. Sci. 112, 14527 (2015).

[76] D. Y. Usachov, A. V. Fedorov, A. E. Petukhov, O. Y. Vilkov, A. G. Rybkin, M. M. Otrokov, A. Arnau, E. V. Chulkov, L. V. Yashina, M. Farjam, V. K. Adamchuk, B. V. Senkovskiy, C. Laubschat, and D. V. Vyalikh, Epitaxial B-Graphene : Large-Scale Growth and Atomic Structure, ACS Nano 9, 7314 (2015).

[77] D. Y. Usachov, A. V. Fedorov, O. Y. Vilkov, A. E. Petukhov, A. G. Rybkin, A. Ernst, M. M. Otrokov, E. V. Chulkov, I. I. Ogorodnikov, M. V. Kuznetsov, L. V. Yashina, E. Y. Kataev, A. V. Erofeevskaya, V. Y. Voroshnin, V. K. Adamchuk, C. Laubschat, and D. V. Vyalikh, Large-Scale Sublattice Asymmetry in Pure and BoronDoped Graphene, Nano Lett. 16, 4535 (2016).

[78] Q. Li, H. Lin, R. Lv, M. Terrones, L. Chi, W. A. Hofer, and M. Pan, Locally Induced Spin States on Graphene by Chemical Attachment of Boron Atoms, Nano Lett. 18, 5482 (2018).

[79] H. González-Herrero, J. M. Gómez-Rodríguez, P. Mallet, M. Moaied, J. J. Palacios, C. Salgado, M. M. Ugeda, J. Y. Veuillen, F. Yndurain, and I. Brihuega, Atomic-scale control of graphene magnetism by using hydrogen atoms, Science (80-. ). 352, 437 (2016), arXiv :1103.3291.

[80] J.-i. Ozaki, T. Anahara, N. Kimura, and A. Oya, Simultaneous doping of boron and nitrogen into a carbon to enhance its oxygen reduction activity in proton exchange membrane fuel cells, Carbon N. Y. 44, 3358 (2006).

[81] S. Wang, E. Iyyamperumal, A. Roy, Y. Xue, D. Yu, and L. Dai, Vertically Aligned BCN Nanotubes as Efficient Metal-Free Electrocatalysts for the Oxygen Reduction Reaction : A Synergetic Effect by Co-Doping with Boron and Nitrogen, Angew. Chemie Int. Ed. 50, 11756 (2011).

[82] Y. Zhao, L. Yang, S. Chen, X. Wang, Y. Ma, Q. Wu, Y. Jiang, W. Qian, and Z. Hu, Can Boron and Nitrogen Co-doping Improve Oxygen Reduction Reaction Activity of Carbon Nanotubes?, J. Am. Chem. Soc. 135, 1201 (2013).

[83] V. V. Ivanovskaya, A. Zobelli, O. Stéphan, P. R. Briddon, and C. Colliex, BN Domains Included into Carbon Nanotubes : Role of Interface, J. Phys. Chem. C 113, 16603 (2009).

[84] Y. Gong, G. Shi, Z. Zhang, W. Zhou, J. Jung, W. Gao, L. Ma, Y. Yang, S. Yang, G. You, R. Vajtai, Q. Xu, A. H. MacDonald, B. I. Yakobson, J. Lou, Z. Liu, and P. M. Ajayan, Direct chemical conversion of graphene to boron- and nitrogen- and carbon-containing atomic layers, Nat. Commun. 5, 3193 (2014).

[85] L. Cheng, J. Meng, X. Pan, Y. Lu, X. Zhang, M. Gao, Z. Yin, D. Wang, Y. Wang, J. You, J. Zhang, and E. Xie, Two-dimensional hexagonal boron-carbon-nitrogen atomic layers, Nanoscale 11, 10454 (2019).

[86] M. Telychko, P. Mutombo, P. Merino, P. Hapala, M. Ondráček, F. C. Bocquet, J. Sforzini, O. Stetsovych, M. Vondráček, P. Jelínek, and M. Švec, Electronic and Chemical Properties of Donor, Acceptor Centers in Graphene, ACS Nano 9, 9180 (2015).

[87] J. Sforzini, M. Telychko, O. Krejčí, M. Vondráček, M. Švec, F. C. Bocquet, and F. S. Tautz, Transformation of metallic boron into substitutional dopants in graphene on 6H- SiC(0001), Phys. Rev. B 93, 041302 (2016).

[88] T. Schiros, D. Nordlund, L. Palova, L. Zhao, M. Levendorf, C. Jaye, D. Reichman, J. Park, M. Hybertsen, and A. Pasupathy, Atomistic Interrogation of B-N Codopant Structures and Their Electronic Effects in Graphene, ACS Nano 10, 6574 (2016).

[89] L. Ci, L. Song, C. Jin, D. Jariwala, D. Wu, Y. Li, A. Srivastava, Z. F. Wang, K. Storr, L. Balicas, F. Liu, and P. M. Ajayan, Atomic layers of hybridized boron nitride and graphene domains, Nat. Mater. 9, 430 (2010).

[90] D. Usachov, O. Vilkov, A. Grüneis, D. Haberer, A. Fedorov, V. K. Adamchuk, A. B. Preobrajenski, P. Dudin, A. Barinov, M. Oehzelt, C. Laubschat, and D. V. Vyalikh, Nitrogen-doped graphene : Efficient growth, structure, and electronic properties, Nano Lett. 11, 5401 (2011), arXiv :arXiv :1208.3129v1.

[91] R. Balog, B. Jørgensen, L. Nilsson, M. Andersen, E. Rienks, M. Bianchi, M. Fanetti, E. Lægsgaard, A. Baraldi, S. Lizzit, Z. Sljivancanin, F. Besenbacher, B. Hammer, T. G. Pedersen, P. Hofmann, and L. Hornekær, Bandgap opening in graphene induced by patterned hydrogen adsorption, Nat. Mater. 9, 315 (2010), arXiv :1006.0589.

[92] R. J. Koch, M. Weser, W. Zhao, F. Viñes, K. Gotterbarm, S. M. Kozlov, O. Höfert, M. Ostler, C. Papp, J. Gebhardt, H. P. Steinrück, A. Görling, and T. Seyller, Growth and electronic structure of nitrogen-doped graphene on Ni(111), Phys. Rev. B - Condens. Matter Mater. Phys. 86, 075401 (2012).

[93] E. Velez-Fort, C. Mathieu, E. Pallecchi, M. Pigneur, M. G. Silly, R. Belkhou, M. Marangolo, A. Shukla, F. Sirotti, and A. Ouerghi, Epitaxial graphene on $4 \mathrm{H}-$ $\mathrm{SiC}(0001)$ grown under nitrogen flux : Evidence of low nitrogen doping and high charge transfer, ACS Nano 6, 10893 (2012).

[94] M. Scardamaglia, C. Struzzi, S. Osella, N. Reckinger, J. F. Colomer, L. Petaccia, R. Snyders, D. Beljonne, and C. Bittencourt, Tuning nitrogen species to control the charge carrier concentration in highly doped graphene, 2D Mater. 3, 011001 (2016).

[95] W. A. de Heer, C. Berger, M. Ruan, M. Sprinkle, X. Li, Y. Hu, B. Zhang, J. Hankinson, and E. Conrad, Large area and structured epitaxial graphene produced by confinement controlled sublimation of silicon carbide, Proc. Natl. Acad. Sci. 108, 16900 (2011), arXiv :1103.3552.

[96] J. Sforzini, P. Hapala, M. Franke, G. Van Straaten, A. Stöhr, S. Link, S. Soubatch, P. Jelínek, T. L. Lee, U. Starke, M. Švec, F. C. Bocquet, and F. S. Tautz, 
Structural and Electronic Properties of Nitrogen-Doped Graphene, Phys. Rev. Lett. 116, 126805 (2016).

[97] J. Gebhardt, R. J. Koch, W. Zhao, O. Höfert, K. Gotterbarm, S. Mammadov, C. Papp, A. Görling, H. P. Steinrück, and T. Seyller, Growth and electronic structure of boron-doped graphene, Phys. Rev. B 87, 155437 (2013).

[98] A. Varykhalov, J. Sánchez-Barriga, A. M. Shikin, C. Biswas, E. Vescovo, A. Rybkin, D. Marchenko, and O. Rader, Electronic and magnetic properties of quasifreestanding graphene on Ni, Phys. Rev. Lett. 101, 157601 (2008).

[99] H. Suzuura and T. Ando, Crossover from Symplectic to Orthogonal Class in a Two-Dimensional Honeycomb Lattice, Phys. Rev. Lett. 89, 266603 (2002).

[100] E. Mccann, K. Kechedzhi, V. I. Fala'Ko, H. Suzuura, T. Ando, and B. L. Altshuler, Weak-localization magnetoresistance and valley symmetry in graphene, Phys. Rev. Lett. 97, 146805 (2006), arXiv :0604015 [condmat].

[101] X. Wu, X. Li, Z. Song, C. Berger, and W. A. De Heer, Weak antilocalization in epitaxial graphene : Evidence for chiral electrons, Phys. Rev. Lett. 98, 136801 (2007), arXiv :0611339 [cond-mat].

[102] F. V. Tikhonenko, D. W. Horsell, R. V. Gorbachev, and A. K. Savchenko, Weak Localization in Graphene Flakes, Phys. Rev. Lett. 100, 056802 (2008).

[103] N. J. G. Couto, D. Costanzo, S. Engels, D.-K. Ki, K. Watanabe, T. Taniguchi, C. Stampfer, F. Guinea, and A. F. Morpurgo, Random Strain Fluctuations as Dominant Disorder Source for High-Quality OnSubstrate Graphene Devices, Phys. Rev. X 4, 041019 (2014).

[104] F. V. Tikhonenko, A. A. Kozikov, A. K. Savchenko, and R. V. Gorbachev, Transition between Electron Localization and Antilocalization in Graphene, Phys. Rev. Lett. 103, 226801 (2009).

[105] G. M. Rutter, J. N. Crain, N. P. Guisinger, T. Li, P. N. First, and J. A. Stroscio, Scattering and interference in epitaxial graphene, Science (80-. ). 317, 219 (2007).

[106] Some disparity exists in the literature. Some authors define a combined time $\tau_{*}^{-1}=\tau_{\text {inter }}^{-1}+\tau_{\text {intra }}^{-1}$ or $\tau_{*}^{-1}=$ $\tau_{\text {inter }}^{-1}+\tau_{\text {intra }}^{-1}+\tau_{w}^{-1}$, where $\tau_{w}$ is associated to trigonal warping $[100,103,110]$; some also define $\tau_{*}$ as the intravalley scattering time [126].

[107] M. Monteverde, C. Ojeda-Aristizabal, R. Weil, K. Bennaceur, M. Ferrier, S. Guéron, C. Glattli, H. Bouchiat, J. N. Fuchs, and D. L. Maslov, Transport and Elastic Scattering Times as Probes of the Nature of Impurity Scattering in Single-Layer and Bilayer Graphene, Phys. Rev. Lett. 104, 126801 (2010).

[108] E. H. Hwang, S. Adam, and S. D. Sarma, Carrier transport in two-dimensional graphene layers, Phys. Rev. Lett. 98, 186806 (2007), arXiv :0610157 [cond-mat].

[109] K. Nomura and A. H. MacDonald, Quantum transport of massless dirac fermions, Phys. Rev. Lett. 98, 076602 (2007), arXiv :0606589 [cond-mat].

[110] J. Moser, H. Tao, S. Roche, F. Alzina, C. M. Sotomayor Torres, and A. Bachtold, Magnetotransport in disordered graphene exposed to ozone : From weak to strong localization, Phys. Rev. B - Condens. Matter Mater. Phys. 81, 205445 (2010), arXiv :1003.1299.

[111] J. Horng, C. F. Chen, B. Geng, C. Girit, Y. Zhang, Z. Hao, H. A. Bechtel, M. Martin, A. Zettl, M. F.
Crommie, Y. R. Shen, and F. Wang, Drude conductivity of Dirac fermions in graphene, Phys. Rev. B - Condens. Matter Mater. Phys. 83, 165113 (2011), arXiv :1007.4623.

[112] M. E. Gershenson, Y. B. Khavin, A. G. Mikhalchuk, H. M. Bozler, and A. L. Bogdanov, Crossover from weak to strong localization in quasi-one-dimensional conductors, Phys. Rev. Lett. 79, 725 (1997).

[113] A. L. Friedman, C. D. Cress, S. W. Schmucker, J. T. Robinson, and O. M. Van'T Erve, Electronic transport and localization in nitrogen-doped graphene devices using hyperthermal ion implantation, Phys. Rev. B 93, 161409 (2016).

[114] X. Li, H. Wang, J. T. Robinson, H. Sanchez, G. Diankov, and H. Dai, Simultaneous nitrogen doping and reduction of graphene oxide, J. Am. Chem. Soc. 131, 15939 (2009), arXiv :0910.0862.

[115] Y. C. Lin, C. Y. Lin, and P. W. Chiu, Controllable graphene N-doping with ammonia plasma, Appl. Phys. Lett. 96, 133110 (2010).

[116] B. Guo, Q. Liu, E. Chen, H. Zhu, L. Fang, and J. R. Gong, Controllable N-doping of graphene, Nano Lett. 10, 4975 (2010), arXiv :1401.2627.

[117] Z. Jin, J. Yao, C. Kittrell, and J. M. Tour, Large-scale growth and characterizations of nitrogen-doped monolayer graphene sheets, ACS Nano 5, 4112 (2011).

[118] Y.-f. Lu, S.-t. Lo, J.-c. Lin, W. Zhang, J.-y. Lu, F.h. Liu, and L. U. E. T. Al, Nitrogen-Doped Graphene Sheets Grown by Chemical Vapor Deposition : Synthesis and In fl uence of Nitrogen Impurities on Carrier Transport, ACS Nano 7, 6522 (2013).

[119] X. Li, J. Zhuang, Y. Sun, J. Bai, Z. Zafar, Z. Ni, B. Jin, and Z. Shi, Enhancement of weak localization for nitrogen-doped graphene by short range potentials, Carbon N. Y. 82, 346 (2015).

[120] M. Rein, N. Richter, K. Parvez, X. Feng, H. Sachdev, M. Kläui, and K. Müllen, Magnetoresistance and charge transport in graphene governed by nitrogen dopants, ACS Nano 9, 1360 (2015).

[121] C. W. Beenakker and H. van Houten, Quantum Transport in Semiconductor Nanostructures, Solid State Phys. - Adv. Res. Appl. 44, 1 (1991), arXiv :0412664 [cond-mat].

[122] Although this theory was shown by $\mathrm{Wu}$ et al. to not fit properly the low field MR of epitaxial graphene [101].

[123] H. C. Wu, M. Abid, Y. C. Wu, C. Ó Coileáin, A. Syrlybekov, J. F. Han, C. L. Heng, H. Liu, M. Abid, and I. Shvets, Enhanced Shubnikov-De Haas Oscillation in Nitrogen-Doped Graphene, ACS Nano 9, 7207 (2015).

[124] D. C. Elias, R. V. Gorbachev, A. S. Mayorov, S. V. Morozov, A. A. Zhukov, P. Blake, L. A. Ponomarenko, I. V. Grigorieva, K. S. Novoselov, F. Guinea, and A. K. Geim, Dirac cones reshaped by interaction effects in suspended graphene, Nat. Phys. 7, 701 (2011).

[125] V. G. Peschanskii and Y. A. Kolesnichenko, On the 60th anniversary of the Lifshitz-Kosevich theory, Low Temp. Phys. 40, 267 (2014).

[126] J. Li, L. Lin, D. Rui, Q. Li, J. Zhang, N. Kang, Y. Zhang, H. Peng, Z. Liu, and H. Q. Xu, Electron-Hole Symmetry Breaking in Charge Transport in Nitrogen-Doped Graphene, ACS Nano 11, 4641 (2017), arXiv :1705.01429.

[127] L. Lin, J. Li, H. Ren, A. L. Koh, N. Kang, H. Peng, H. Q. $\mathrm{Xu}$, and Z. Liu, Surface Engineering of Copper Foils for 
Growing Centimeter-Sized Single-Crystalline Graphene, ACS Nano 10, 2922 (2016).

[128] L. Lin, J. Li, Q. Yuan, Q. Li, J. Zhang, L. Sun, D. Rui, Z. Chen, K. Jia, M. Wang, Y. Zhang, M. H. Rummeli, N. Kang, H. Q. Xu, F. Ding, H. Peng, and Z. Liu, Nitrogen cluster doping for high-mobility/conductivity graphene films with millimeter-sized domains, Sci. Adv. 5, eaaw8337 (2019).

[129] Z. M. Ao, Q. Jiang, R. Q. Zhang, T. T. Tan, and S. Li, Al doped graphene : A promising material for hydrogen storage at room temperature, J. Appl. Phys. 105, 74307 (2009).

[130] A. S. Rad, Al-doped graphene as a new nanostructure adsorbent for some halomethane compounds : DFT calculations, Surf. Sci. 645, 6 (2016).

[131] J. Albero, A. Vidal, A. Migani, P. Concepción, L. Blancafort, and H. García, Phosphorus-Doped Graphene as a Metal-Free Material for Thermochemical Water Reforming at Unusually Mild Conditions, ACS Sustain. Chem. Eng. 7, 838 (2019).

[132] K. Pi, K. M. McCreary, W. Bao, W. Han, Y. F. Chiang, Y. Li, S.-W. Tsai, C. N. Lau, and R. K. Kawakami, Electronic doping and scattering by transition metals on graphene, Phys. Rev. B 80, 75406 (2009).

[133] A. V. Krasheninnikov, P. O. Lehtinen, A. S. Foster, P. Pyykkö, and R. M. Nieminen, Embedding TransitionMetal Atoms in Graphene : Structure, Bonding, and Magnetism, Phys. Rev. Lett. 102, 126807 (2009).

[134] M. Kaukonen, A. V. Krasheninnikov, E. Kauppinen, and R. M. Nieminen, Doped Graphene as a Material for Oxygen Reduction Reaction in Hydrogen Fuel Cells : A Computational Study, ACS Catal. 3, 159 (2013).

[135] X. Mao, G. Kour, C. Yan, Z. Zhu, and A. Du, Single Transition Metal Atom-Doped Graphene Supported on a Nickel Substrate : Enhanced Oxygen Reduction Reactions Modulated by Electron Coupling, J. Phys. Chem. C 123, 3703 (2019).

[136] W. Wan, H. Li, H. Huang, S. L. Wong, L. Lv, Y. Gao, and A. T. S. Wee, Incorporating Isolated Molybdenum (Mo) Atoms into Bilayer Epitaxial Graphene on 4HSiC(0001), ACS Nano 8, 970 (2014).

[137] V. Carnevali, L. L. Patera, G. Prandini, M. Jugovac, S. Modesti, G. Comelli, M. Peressi, and C. Africh, Do- ping of epitaxial graphene by direct incorporation of nickel adatoms, Nanoscale 11, 10358 (2019).

[138] C. R. Dean, A. F. Young, I. Meric, C. Lee, L. Wang, S. Sorgenfrei, K. Watanabe, T. Taniguchi, P. Kim, K. L. Shepard, and J. Hone, Boron nitride substrates for highquality graphene electronics, Nat. Nanotechnol. 5, 722 (2010).

[139] R. Decker, Y. Wang, V. W. Brar, W. Regan, H.-Z. Tsai, Q. Wu, W. Gannett, A. Zettl, and M. F. Crommie, Local Electronic Properties of Graphene on a BN Substrate via Scanning Tunneling Microscopy, Nano Lett. 11, 2291 (2011).

[140] L. Banszerus, M. Schmitz, S. Engels, J. Dauber, M. Oellers, F. Haupt, K. Watanabe, T. Taniguchi, B. Beschoten, and C. Stampfer, Ultrahigh-mobility graphene devices from chemical vapor deposition on reusable copper, Sci. Adv. 1 (2015).

[141] Z. Wang, S. Qin, and C. Wang, Electronic and magnetic properties of single-layer graphene doped by nitrogen atoms, Eur. Phys. J. B 87, 88 (2014).

[142] R. Babar and M. Kabir, Ferromagnetism in nitrogendoped graphene, Phys. Rev. B 99, 115442 (2019).

[143] P. Błoński, J. Tuček, Z. Sofer, V. Mazánek, M. Petr, M. Pumera, M. Otyepka, and R. Zbořil, Doping with Graphitic Nitrogen Triggers Ferromagnetism in Graphene, J. Am. Chem. Soc. 139, 3171 (2017).

[144] J. Tuček, P. Błoński, Z. Sofer, P. Šimek, M. Petr, M. Pumera, M. Otyepka, and R. Zbořil, Sulfur Doping Induces Strong Ferromagnetic Ordering in Graphene : Effect of Concentration and Substitution Mechanism, Adv. Mater. 28, 5045 (2016).

[145] M. Zhu, C. Cao, J. Chen, Y. Sun, R. Ye, J. Xu, and Y.-F. Han, Electronic Tuning of Cobalt Porphyrins Immobilized on Nitrogen-Doped Graphene for CO2 Reduction, ACS Appl. Energy Mater. 2, 2435 (2019).

[146] H. Fei, J. Dong, M. J. Arellano-Jiménez, G. Ye, N. Dong Kim, E. L. G. Samuel, Z. Peng, Z. Zhu, F. Qin, J. Bao, M. J. Yacaman, P. M. Ajayan, D. Chen, and J. M. Tour, Atomic cobalt on nitrogen-doped graphene for hydrogen generation, Nat. Commun. 6, 8668 (2015). 